\title{
The $\mathrm{C}_{60}: \mathrm{C}_{60}^{+}$ratio in diffuse and translucent interstellar clouds
}

\author{
Gaël Rouillé ${ }^{1}$, Serge A. Krasnokutski ${ }^{1} \odot$, and Yvain Carpentier ${ }^{1,2}$ \\ ${ }^{1}$ Laboratory Astrophysics Group of the Max Planck Institute for Astronomy at the Friedrich Schiller University Jena, Institute of \\ Solid State Physics, Helmholtzweg 3, 07743 Jena, Germany \\ e-mail: gael.rouille@uni-jena.de \\ ${ }^{2}$ University of Lille, CNRS, UMR 8523 - PhLAM - Laboratoire de Physique des Lasers Atomes et Molécules, 59000 Lille, France
}

Received 24 August 2021 / Accepted 12 September 2021

\begin{abstract}
Context. Insight into the conditions that drive the physics and chemistry in interstellar clouds is gained from determining the abundance and charge state of their components.

Aims. We propose an evaluation of the $\mathrm{C}_{60}: \mathrm{C}_{60}^{+}$ratio in diffuse and translucent interstellar clouds that exploits electronic absorption bands so as not to rely on ambiguous IR emission measurements.

Methods. The ratio is determined by analyzing archival spectra and literature data. Information on the cation population is obtained from published characteristics of the main diffuse interstellar bands attributed to $\mathrm{C}_{60}^{+}$and absorption cross sections already reported for the vibronic bands of the cation. The population of neutral molecules is described in terms of upper limits because the relevant vibronic bands of $\mathrm{C}_{60}$ are not brought out by observations. We revise the oscillator strengths reported for $\mathrm{C}_{60}$ and measure the spectrum of the molecule isolated in $\mathrm{Ne}$ ice to complete them.

Results. We scale down the oscillator strengths for absorption bands of $\mathrm{C}_{60}$ and find an upper limit of approximately 1.3 for the $\mathrm{C}_{60}: \mathrm{C}_{60}^{+}$ ratio.

Conclusions. We conclude that the fraction of neutral molecules in the buckminsterfullerene population of diffuse and translucent interstellar clouds may be notable despite the non-detection of the expected vibronic bands. More certainty will require improved laboratory data and observations.
\end{abstract}

Key words. astrochemistry - ISM: abundances - ISM: lines and bands - ISM: molecules

\section{Introduction}

Mid-IR emission observations revealed the presence of fullerene $\mathrm{C}_{60}$ first in planetary and reflection nebulae (Cami et al. 2010; Sellgren et al. 2010) and then in various astrophysical objects (Cami 2014, and references therein). More recently, also exploiting mid-IR emission observations, Berné et al. (2017) found that $\mathrm{C}_{60}$ is present in the diffuse interstellar medium (ISM) as well. As to the fullerene cation $\mathrm{C}_{60}^{+}$, after two decades of anticipation (Foing \& Ehrenfreund 1994, 1997), it was identified recently as the carrier of diffuse interstellar bands (DIBs) observed at nearIR wavelengths (Campbell et al. 2015, 2016a,b; Walker et al. 2015, 2016, 2017). After several tests, the identification of $\mathrm{C}_{60}^{+}$ as the carrier is considered to be firm (Cordiner et al. 2019, and references therein), demonstrating the presence of $\mathrm{C}_{60}^{+}$in the diffuse ISM. Lastly, Iglesias-Groth (2019) observed interstellar fullerenes $\mathrm{C}_{60}$ and $\mathrm{C}_{70}$, and the ions $\mathrm{C}_{60}^{+}$and $\mathrm{C}_{60}^{-}$, in IR emission spectra of the star-forming region IC 348.

The ubiquity of $\mathrm{C}_{60}$ fullerene makes it an attractive species for probing various astrophysical environments (Brieva et al. 2016, and references therein). As an example of application, the $\mathrm{C}_{60}: \mathrm{C}_{60}^{+}$ratio in the ISM gives information on the local interstellar radiation field (ISRF) and electron density since it relates to photoionization and electron recombination (Bakes \& Tielens 1995). Berné et al. (2017) evaluated the $\mathrm{C}_{60}: \mathrm{C}_{60}^{+}$ratio for the diffuse ISM and, from the observed mid-IR emission bands of $\mathrm{C}_{60}$ and the measurement of DIBs attributed to $\mathrm{C}_{60}^{+}$, they obtained absolute abundances that point to a $\mathrm{C}_{60}: \mathrm{C}_{60}^{+}$ratio between 0.3 and 6. Thus, the neutral species is possibly more abundant than the cation in the diffuse ISM, in agreement with a model predicting $\mathrm{C}_{60}: \mathrm{C}_{60}^{+}: \mathrm{C}_{60}^{-}$relative abundances of 0.62:0.11:0.27 (estimated from Fig. 5 in Bakes \& Tielens 1995), that is, a $\mathrm{C}_{60}: \mathrm{C}_{60}^{+}$ratio of 5.6. The large uncertainty that affects the ratio given by Berné et al. (2017) originates chiefly in the evaluation of the column density of the neutral molecule from measurements of its IR emission spectrum. Even though improved emission observations may be a remedy, another issue arises. Indeed, the IR emission bands attributed to $\mathrm{C}_{60}$ may actually comprise contributions by analogous species (García-Hernández et al. 2013; Krasnokutski et al. 2019).

Electronic absorption bands are, by contrast, specific and better suited for probing the diffuse ISM, hence attempts to exploit those of $\mathrm{C}_{60}$ to evaluate the $\mathrm{C}_{60}: \mathrm{C}_{60}^{+}$ratio. Resulting ratio values range from 1 (Léger et al. 1988) down to upper limits of 0.1 (Maier \& Campbell 2017) and 0.0075 (Herbig 2000), the last two indicating strongly ionized populations, in contradiction with the prediction by Bakes \& Tielens (1995) and the range of values proposed by Berné et al. (2017).

Facing this discrepancy, we propose another determination of the $\mathrm{C}_{60}: \mathrm{C}_{60}^{+}$ratio in the diffuse ISM. Information on the cation population is provided to us by published detailed measurements of DIBs that $\mathrm{C}_{60}^{+}$carries. As to the population of neutral buckminsterfullerene, it is characterized by an upper limit because the absorption bands of $\mathrm{C}_{60}$ that are most promising as gauges, expected at wavelengths near 4000 and $6000 \AA$, do not appear in the astronomical spectra at hand. Thus, we calculate an upper 
Table 1. Column densities of $\mathrm{C}_{60}^{+}$.

\begin{tabular}{llllll}
\hline \hline LOS & $\lambda(\AA)$ & $w(\AA)$ & $W(\mathrm{~m})$ & $N\left(10^{12} \mathrm{~cm}^{-2}\right)$ & $\Delta N\left(10^{12} \mathrm{~cm}^{-2}\right)$ \\
\hline HD 76341 & $9577.2(0.2)$ & $2.5(0.2)$ & $110(20)$ & 10.5 & 6.5 \\
& $9632.2(0.2)$ & $2.5(0.2)$ & $134(30)$ & 18 & 12 \\
HD 136239 & $9576.9(0.2)$ & $2.9(0.2)$ & $195(15)$ & 19 & 11 \\
& $9631.8(0.1)$ & $2.4(0.2)$ & $120(20)$ & 16 & 10 \\
HD 148379 & $9577.3(0.2)$ & $3.3(0.2)$ & $137(9)$ & 13.2 & 7.8 \\
& $9632.2(0.2)$ & $2.4(0.2)$ & $80(11)$ & 10.8 & 6.6 \\
HD 167264 & $9576.8(0.2)$ & $3.2(0.2)$ & $60(17)$ & 5.7 & 3.8 \\
& $9632.4(0.2)$ & $2.7(0.2)$ & $82(20)$ & 11.1 & 7.2 \\
HD 168625 & $9576.2(0.1)$ & $3.1(0.2)$ & $320(25)$ & 32 & 19 \\
& $9631.5(0.1)$ & $1.8(0.1)$ & $194(25)$ & 27 & 16 \\
HD 169454 & $9577.1(0.1)$ & $2.3(0.2)$ & $82(10)$ & $7.8^{(a)}$ & 4.7 \\
& $9631.4(0.3)$ & $2.7(0.2)$ & $130(20)$ & 18 & 11 \\
HD 183143 & $9577.3(0.2)$ & $2.9(0.2)$ & $300(20)$ & $30^{(a),(b)}$ & 18 \\
& $9632.5(0.2)$ & $1.9(0.3)$ & $105(20)$ & 14.3 & 9.0 \\
\hline
\end{tabular}

Notes. The values of $\lambda, w$, and $W$ are from Galazutdinov et al. (2017). Values between parentheses are measurement errors. ${ }^{(a)}$ Consistent with $9 \times 10^{12} \mathrm{~cm}^{-2}$ toward HD 169454 and $20 \times 10^{12} \mathrm{~cm}^{-2}$ toward HD 183143 in Berné et al. (2017). ${ }^{(b)}$ Consistent with $2 \times 10^{13} \mathrm{~cm}^{-2}$ in Walker et al. (2015) and $(2 \pm 0.8) \times 10^{13} \mathrm{~cm}^{-2}$ in Campbell et al. (2016b).

limit for $\mathrm{C}_{60}: \mathrm{C}_{60}^{+}$along lines of sight (LOSs) for which spectra of the 4000 and $6000 \AA$ regions are available and for which the DIBs carried by $\mathrm{C}_{60}^{+}$are documented in detail. For this purpose, we evaluate anew the oscillator strength of the relevant bands in the electronic spectrum of $\mathrm{C}_{60}$.

\section{Lines of sight}

We examined seven LOSs chosen for the availability of detailed measurements of the two strongest DIBs attributed to $\mathrm{C}_{60}^{+}$ (Galazutdinov et al. 2017) and that of spectra that cover the 4000 and $6000 \AA$ wavelength regions, where absorption bands of $\mathrm{C}_{60}$ are expected. These LOSs exhibit a color excess $E(B-V)$ in the range $0.2-1.48$, which translates approximately into total extinction $A_{\mathrm{V}}$ values in the range 0.6-4.6 if we adopt the totalto-selective extinction ratio of the Milky Way $\left(R_{\mathrm{V}}=3.1\right.$, Cardelli et al. 1989). Although these values suggest the LOSs cross diffuse $\left(A_{\mathrm{V}} \lesssim 1\right)$ to translucent $\left(1 \lesssim A_{\mathrm{V}} \lesssim 5\right)$ clouds, one must not presume the distribution of matter along them. Indeed, an alignment of diffuse clouds may not differ from a translucent cloud in terms of extinction. In any case, the spectra of all the corresponding target stars feature lines of the $\mathrm{CH}$ radical, indicating regions with diffuse molecular cloud conditions (Snow \& McCall 2006). Furthermore, all except one include lines of the $\mathrm{CN}$ radical. As to translucent cloud conditions, they are actually met in at least one case, toward HD 169454 (Jannuzi et al. 1988).

The measurements of the 9577 and $9632 \AA$ DIBs by Galazutdinov et al. (2017) along the seven LOSs we have chosen are given in Table 1. They comprise the observed rest wavelengths, $\lambda$, full widths at half maximum (FWHMs), $w$, and equivalent widths, $W$.

Spectra of the LOS target stars measured in the 4000 and $6000 \AA$ wavelength regions with a high signal-to-noise ratio $(\mathrm{S} / \mathrm{N})$ were found in the archives of the European Southern Observatory (ESO). For each LOS, spectra measured in series in the same observational session were summed to further increase the $\mathrm{S} / \mathrm{N}$. The errors on the flux values were then considered to be standard errors and were combined accordingly. The data sets are given in Appendix A.
The wavelength scales of the archival spectra are defined in the observer rest frame. For practical purposes, the standard of rest has been changed to an interstellar reference, a substance plausibly located in the same regions as interstellar fullerenes, so as to obtain scales that show the rest wavelengths of these species (Appendix B).

\section{Laboratory spectra of $\mathrm{C}_{60}$ and $\mathrm{C}_{60}^{+}$}

\subsection{Positions and widths of the near-IR bands of $C_{60}^{+}$}

Campbell et al. (2016b) measured the near-IR spectrum of the $\mathrm{C}_{60}^{+} \cdot \mathrm{He}$ complex in the gas phase below $10 \mathrm{~K}$. They found that absorption bands of free $\mathrm{C}_{60}^{+}$were similar in terms of wavelengths and relative intensities to those obtained for the complex, and that they matched DIBs as a consequence, leading to the first identification of one of their carriers (Cordiner et al. 2019, and references therein). We are presently interested in the two strongest bands of $\mathrm{C}_{60}^{+}$, which absorb at $9577.5 \pm 0.1 \AA$ and $9632.7 \pm 0.1 \AA$ according to Campbell et al. (2016b).

Exploiting the spectrum of $\mathrm{C}_{60}^{+} \cdot \mathrm{He}$ further, Campbell et al. (2016b) determined the FWHMs of these two bands of $\mathrm{C}_{60}^{+}$for a temperature of $10 \mathrm{~K}$. Denoted $w\left(\mathrm{C}_{60}^{+}, 9577.5 \AA\right)$ and $w\left(\mathrm{C}_{60}^{+}\right.$, $9632.7 \AA$ ), they are $2.5 \pm 0.2 \AA$ and $2.2 \pm 0.2 \AA$, respectively.

\subsection{Positions and widths of the near-UV and orange bands of $C_{60}$}

Measurements in a molecular beam with resonant two-photon ionization spectroscopy showed that the $1^{1} \mathrm{~T}_{1 u} \leftarrow{ }^{1} \mathrm{~A}_{g}$ transition of $\mathrm{C}_{60}$ gives narrow absorption bands near $4000 \AA$ (Haufler et al. 1991). The two strongest bands, labeled $A_{0}$ and $A_{1}$ to follow Leach et al. (1992), were thoroughly examined by Sassara et al. (2001) to assist in searches for interstellar $\mathrm{C}_{60}$. They arise respectively at $4024 \pm 0.5 \AA$ and $3980 \pm 0.5 \AA$, and, in interstellar conditions, the FWHMs $w\left(\mathrm{C}_{60}, \mathrm{~A}_{0}\right)$ and $w\left(\mathrm{C}_{60}, \mathrm{~A}_{1}\right)$ would be $4.05 \pm 0.81$ and $5.54 \pm 0.80 \AA$, respectively (or $25 \pm 5$ and $35 \pm 5 \mathrm{~cm}^{-1}$ in terms of wavenumbers in Sassara et al. 2001). 
G. Rouillé et al.: The $\mathrm{C}_{60}: \mathrm{C}_{60}^{+}$ratio in diffuse and translucent interstellar clouds

The absorption bands of $\mathrm{C}_{60}$ near $6000 \AA$ have not been examined with the attention given those near $4000 \AA$, possibly because they originate in electric dipole-forbidden electronic transitions (Gasyna et al. 1991; Leach et al. 1992; Negri et al. 1992; Hansen et al. 1997; Orlandi \& Negri 2002) and, hence, are expected to be weaker than the near-UV bands. Groundbased astronomical measurements, however, when atmospheric absorptions are avoided, are more effective in terms of sensitivity and resolution in the $6000 \AA$ wavelength region than near $4000 \AA$. It is thus worthwhile to examine and characterize the orange bands of $\mathrm{C}_{60}$.

The assignment of the bands observed near $6000 \AA$ is complex because three electronic transitions are involved (Orlandi \& Negri 2002, and references therein). The bands were measured over a broad wavelength domain with $\mathrm{C}_{60}$ isolated in $\mathrm{Ar}$ ice at $\sim 5 \mathrm{~K}$ (Gasyna et al. 1991) and, with greater detail, in Ne ice at $4 \mathrm{~K}$ (Sassara et al. 1997) and in He droplets at 0.37 K (Close et al. 1997). Measurements were also performed with $\mathrm{C}_{60}$ in hydrocarbon solvents at room temperature and at $77 \mathrm{~K}$ (Leach et al. 1992; Hora et al. 1996; Catálan \& Pérez 2002). Prominent bands appeared to form a series, and the member at longest wavelength was labeled $\gamma_{0}$ by Leach et al. (1992).

In a solvatochromism study, the $\gamma_{0}$ band was predicted to arise at $607.3 \pm 0.2 \mathrm{~nm}$ in the gas phase. In another such study, Renge (1995) observed the influence of temperature on the extrapolated gas-phase wavelength and proposed a transition energy of $16482 \pm 24 \mathrm{~cm}^{-1}$ for the $\gamma_{0}$ band of cold $\mathrm{C}_{60}$ in the gas phase, which translates into a wavelength of $606.7 \pm 0.9 \mathrm{~nm}$. Finally, the spectrum of $\mathrm{C}_{60}$ isolated in $\mathrm{He}$ droplets, least affected by environment-induced shifts, yielded a wavelength of $607.17 \mathrm{~nm}$ (Close et al. 1997).

Haufler et al. (1991) had already observed narrow bands near $6000 \AA$ in their study of $\mathrm{C}_{60}$ in a molecular beam, the strongest one rising near $6070 \AA$. Catálan (1994) identified this band with $\gamma_{0}$, the assignment of which is still uncertain (Orlandi \& Negri 2002, and references therein).

We presently determine a wavelength of $6070 \pm 1.0 \AA$ for the $\gamma_{0}$ band from the examination of the photoionization spectrum (Fig. 2 in Haufler et al. 1991). It is consistent with the values extrapolated using solvatochromism, the value reported for $\mathrm{C}_{60}$ in He droplets, and particularly a later molecular-beam measurement (Hansen et al. 1997). The uncertainty does not include that of the spectrometer calibration, which was not indicated.

Haufler et al. (1991) remarked that the FWHM of some bands was less than $5 \mathrm{~cm}^{-1}$, which we find correct in the case of the band at $6070 \AA$ A. Consequently, assuming the FWHM of the absorption band is identical to that of the measured photoionization feature, we set $w\left(\mathbf{C}_{60}, \gamma_{0}\right)$ to $1.5 \pm 0.5 \AA$ in the cold gas phase. The temperature of the molecules in the molecular beam is uncertain, yet it is likely closer to 100 than $10 \mathrm{~K}$ because the vaporization process provides the molecules with much internal energy and cooling them in a carrier gas expansion becomes less effective as their mass increases. Nevertheless, the temperature may not be higher than $100 \mathrm{~K}$ in the case of $\mathrm{C}_{60}$ in a beam as estimated by Hansen et al. (1997). This is also suggested by the comparison of the FWHM presently adopted with that of simulated rotational contours (Edwards \& Leach 1993). At $1.5 \pm 0.5 \AA$, equivalent to $4.1 \pm 1.4 \mathrm{~cm}^{-1}$, the FWHM and its uncertainty indicate temperatures between 50 and $100 \mathrm{~K}$, such as those encountered in the less dense regions of diffuse molecular clouds. At the lower temperatures found in the denser regions of diffuse clouds and in translucent clouds, the FWHM would be smaller according to the simulations.

\subsection{Oscillator strengths of the near-IR bands of $C_{60}^{+}$}

Campbell et al. (2016b) derived the absorption cross sections of the bands that arise at 9577.5 and $9632.7 \AA$ in the spectrum of $\mathrm{C}_{60}^{+}$. They are denoted $\sigma\left(\mathrm{C}_{60}^{+}, 9577.5 \AA\right)$ and $\sigma\left(\mathrm{C}_{60}^{+}\right.$, $9632.7 \AA)$ and their respective values are $(5 \pm 2) \times 10^{-15} \mathrm{~cm}^{2}$ and $(4 \pm 1.6) \times 10^{-15} \mathrm{~cm}^{2}$.

From their observational spectra, Galazutdinov et al. (2017) derived FWHMs for the two strongest DIBs attributed to $\mathrm{C}_{60}^{+}$, $w(\mathrm{DIB}, 9577 \AA)$ ranging from $2.3 \pm 0.2$ to $3.3 \pm 0.2 \AA$, and $w(\mathrm{DIB}, 9632 \AA$ ) ranging from $1.8 \pm 0.1$ to $2.7 \pm 0.2 \AA$. The deviation toward values larger than the laboratory measurements can be attributed to a higher temperature of the interstellar ions compared with the $\mathrm{C}_{60}^{+} \cdot \mathrm{He}$ complexes. It can also be attributed, at least partially, to the quality of the observational spectra. Indeed, assuming the two DIBs are caused by the same carrier, their widths should vary consistently from an LOS to another, when they do not. Moreover, deviations toward smaller values are not expected. Consequently, we assign the experimental absorption cross sections to the DIBs without considering any correction derived from their widths.

Spectra of $\mathrm{C}_{60}^{+}$isolated in $\mathrm{Ne}$ ice were also reported and, as expected, the bands arising at 9577.5 and $9632.7 \AA$ for the free cation are then slightly redshifted (Fulara et al. 1993; Strelnikov et al. 2015). Strelnikov et al. (2015) attributed oscillator strengths of $0.01 \pm 0.003$ and $0.015 \pm 0.005$ to the bluer and redder bands, respectively. Thus, the relative intensities are reversed compared to gas-phase measurements, an effect one may attribute to the interaction between the cations and the Ne matrix, unless the order of the bands itself is reversed, which would be unexpected.

We can also compute effective oscillator strengths in a straightforward manner from the absorption cross sections and widths determined by Campbell et al. (2016b) through

$f=\frac{4 \epsilon_{0} m c^{2}}{e^{2}} \frac{\sigma w_{v}}{k}$

with

$k=2 \sqrt{\frac{\ln 2}{\pi}}$,

where $m$ and $e$ are the mass and charge of an electron, and $c$ is the speed of light in vacuum. Equation (1) is obtained by making the approximation that band profiles are Gaussian in the wavenumber domain, and by using such a profile with FWHM $w_{v}$ when formulating the oscillator strength (Mulliken 1939, and references therein). In order to use $w$ from the wavelength domain as given in Sect. 3.1, Eq. (1) can be approximated with

$f=\frac{4 \epsilon_{0} m c^{2}}{e^{2} \lambda^{2}} \frac{\sigma w}{k}$.

Values of $0.0164 \pm 0.008$ and $0.0114 \pm 0.006$ are obtained for $f\left(\mathrm{C}_{60}^{+}, 9577.5 \AA\right)$ and $f\left(\mathrm{C}_{60}^{+}, 9632.7 \AA\right)$, respectively. The reversal aside, they are consistent with those derived by Strelnikov et al. (2015). We retain the strengths derived from the absorption cross sections measured by Campbell et al. (2016b) on the $\mathrm{C}_{60}^{+} \cdot \mathrm{He}$ complex because the spectrum of $\mathrm{C}_{60}^{+}$is less affected by the interaction with a single $\mathrm{He}$ atom than by the interaction with several $\mathrm{Ne}$ atoms, and because the gas-phase spectrum of the $\mathrm{C}_{60}^{+} \cdot \mathrm{He}$ complex is in better agreement with the DIB spectrum in terms of relative strengths compared to the matrix-isolation measurements. 


\subsection{Oscillator strengths of the near-UV and orange bands of $\mathrm{C}_{60}$}

Haufler et al. (1991) determined an oscillator strength of $0.006 \pm 0.002$ for the peaks of the $1^{1} \mathrm{~T}_{1 u} \leftarrow{ }^{1} \mathrm{~A}_{g}$ transition, namely, the system of $A_{n}$ bands of Leach et al. (1992). They did so by comparing their spectrum of $\mathrm{C}_{60}$ in a molecular beam with a spectrum obtained from a solution. Yet, from the spectrum of $\mathrm{C}_{60}$ in hexane at room temperature, Leach et al. (1992) obtained a value of $0.015 \pm 0.005$ for the same transition. The discrepancy between the two values possibly originates in the fact that Leach et al. (1992) did not correct the oscillator strength for the effect of the solvent (Chupka \& Klots 1997). Considering a refractive index of $1.39 \pm 0.01$ for hexane at room temperature at $400 \mathrm{~nm}$ (estimated from Fig. 3 in Sowers et al. 1972), a correction factor of $0.582 \pm 0.008$ is obtained, which leads to an oscillator strength of $0.0087 \pm 0.0031$ for the $1^{1} \mathrm{~T}_{1 u} \leftarrow{ }^{1} \mathrm{~A}_{g}$ transition of free $\mathrm{C}_{60}$. This value is in good agreement with that reported by Haufler et al. (1991), which is consequently chosen for our study.

We determined the oscillator strengths of the $A_{0}$ and $A_{1}$ bands by distributing the value given by Haufler et al. (1991) among the narrow peaks measured in a spectrum of $\mathrm{C}_{60}$ isolated in $\mathrm{Ne}$ ice, in proportion to their areas, which we assume to be similarly affected by the matrix environment. The spectroscopy is briefly described in Appendix C. Figure 1 shows the matrix-isolation spectrum and Gaussian profiles fitted onto seven peaks of the $1^{1} \mathrm{~T}_{1 u} \leftarrow{ }^{1} \mathrm{~A}_{g}$ transition, that is, the system of $\mathrm{A}_{n}$ bands. The fitting procedure gave for the seven peaks together an area of $6.64 \pm 0.21 \mathrm{~cm}^{-1}$, with individual peak areas of $1.386 \pm 0.064 \mathrm{~cm}^{-1}$ for $\mathrm{A}_{0}$ and $2.938 \pm 0.073 \mathrm{~cm}^{-1}$ for $\mathrm{A}_{1}$. Thus, values of $0.00131 \pm 0.00056$ and $0.0027 \pm 0.0011$ were derived for $f\left(\mathrm{C}_{60}, \mathrm{~A}_{0}\right)$ and $f\left(\mathrm{C}_{60}, \mathrm{~A}_{1}\right)$, respectively. They are visibly lower than those used by Berné et al. (2017), 0.005 and 0.007 , yet on the same order.

Similarly, the oscillator strength of the orange band $\gamma_{0}$ was obtained by comparing its area of $0.435 \pm 0.022 \mathrm{~cm}^{-1}$ with that of the $\mathrm{A}_{1}$ band (Fig. 1). Consequently, $f\left(\mathrm{C}_{60}, \gamma_{0}\right)$ equals $0.00040 \pm 0.00016$.

\section{Astronomical spectra}

\subsection{Observational data near $4000 \AA$}

Several atomic lines arise in stellar spectra around $4000 \AA$. The strongest lines are the $\mathrm{HI}(\mathrm{H} \epsilon)$ and $\mathrm{He}$ I lines at 3970.075 and 4026.1914 A rest wavelengths, respectively. While the H I and He I lines are observed in all spectra, the others appear with relative strengths that vary widely depending on the target star.

In each observational spectrum taken into account, the $\mathrm{A}_{0}$ band of $\mathrm{C}_{60}$ expected at $4024 \AA$ would overlap the strong $\mathrm{He} \mathrm{I}$ line mentioned above. Figure 2 illustrates the phenomenon in the spectrum of HD 169454; it also shows that the $\mathrm{A}_{0}$ band and a minor He I line (4023.973 $\AA$ rest wavelength) are superimposed, not to mention the overlap by an Fe III line (4022.35 rest wavelength, Thompson et al. 2008, and references therein).

In comparison, the $\mathrm{A}_{1}$ band of $\mathrm{C}_{60}$ at $3980 \AA$ is better separated from the nearby O II line found at $3982.7140 \AA$ rest wavelength, and from S III lines that rise at longer wavelengths. It is superimposed with a line of S II (3979.829 $\AA$ rest wavelength), which is apparent toward HD 183143 as observed in Fig. 3. Figure 3 also shows that the strength of the O II, S II, and $\mathrm{S}$ III lines depends on the spectral type of the target star and can be extremely weak.
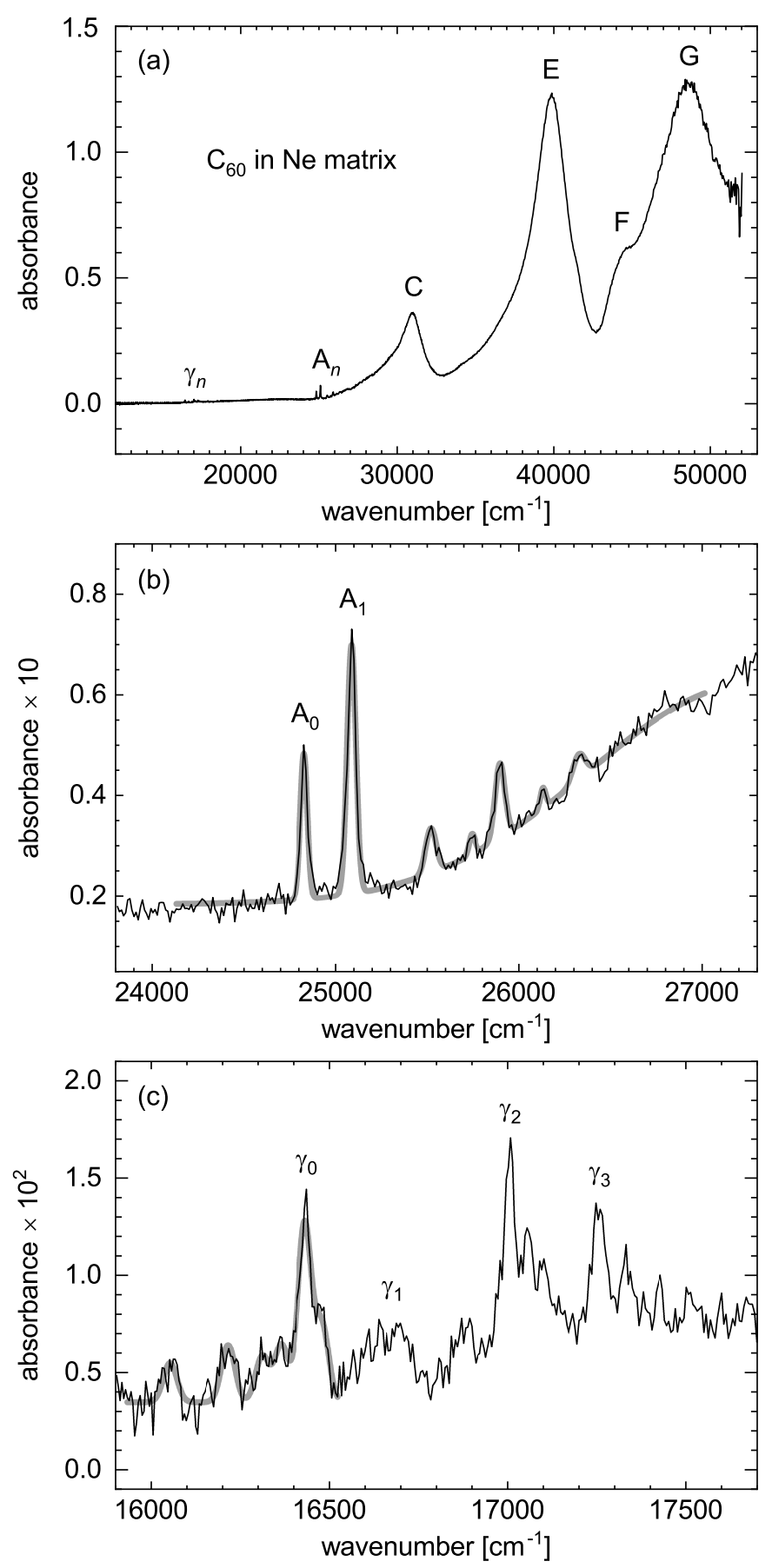

Fig. 1. (a) Absorption spectrum of $\mathrm{C}_{60}$ isolated in a $\mathrm{Ne}$ matrix, not corrected for scattering. Band labels according to Leach et al. (1992). (b) Gaussian profiles (thick gray solid curve) fitted to bands of the $1^{1} \mathrm{~T}_{1 u} \leftarrow{ }^{1} \mathrm{~A}_{g}$ transition, including $\mathrm{A}_{0}$ and $\mathrm{A}_{1}$, and to the baseline. (c) Gaussian profiles (thick gray solid curve) fitted, with a common FWHM, to absorptions near the $\gamma_{0}$ band. Fitted band positions are $16431.1 \pm 1.2 \mathrm{~cm}^{-1}, 24829.04 \pm 0.98 \mathrm{~cm}^{-1}$, and $25087.73 \pm 0.65 \mathrm{~cm}^{-1}$ for $\gamma_{0}, A_{0}$, and $A_{1}$, respectively. The wavelength scale of the spectrometer was calibrated with an accuracy of $0.3 \mathrm{~nm}$.

Overlap with stellar lines is critical when the interstellar band is comparatively much weaker or, a fortiori, not observed because it lowers the $\mathrm{S} / \mathrm{N}$ that is taken into account to determine the column density of the interstellar species or its upper limit. In such an instance, concerning the $4000 \AA$ region, the $\mathrm{A}_{1}$ band of $\mathrm{C}_{60}$ at $3980 \AA$ is preferred to $\mathrm{A}_{0}$, although broadening of the $\mathrm{H} \epsilon$ 
G. Rouillé et al.: The $\mathrm{C}_{60}: \mathrm{C}_{60}^{+}$ratio in diffuse and translucent interstellar clouds

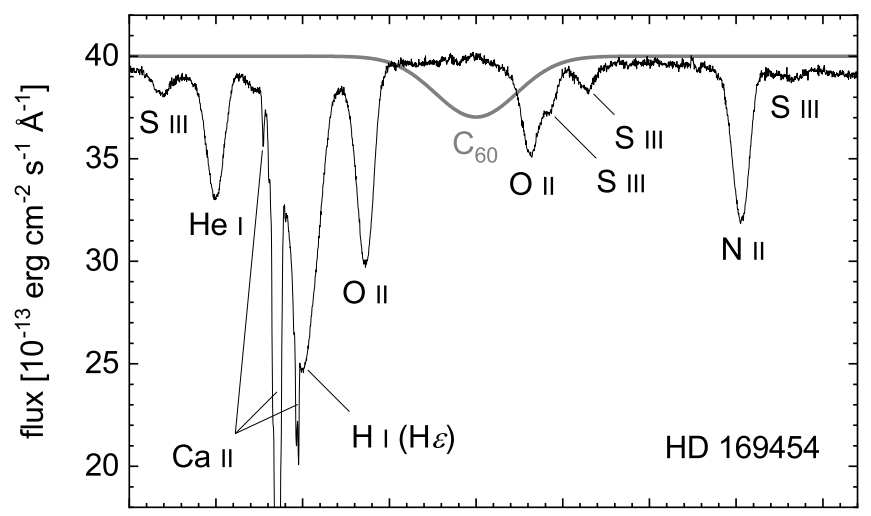

396039653970397539803985399039954000

interstellar diatomics rest wavelength $[\AA]$

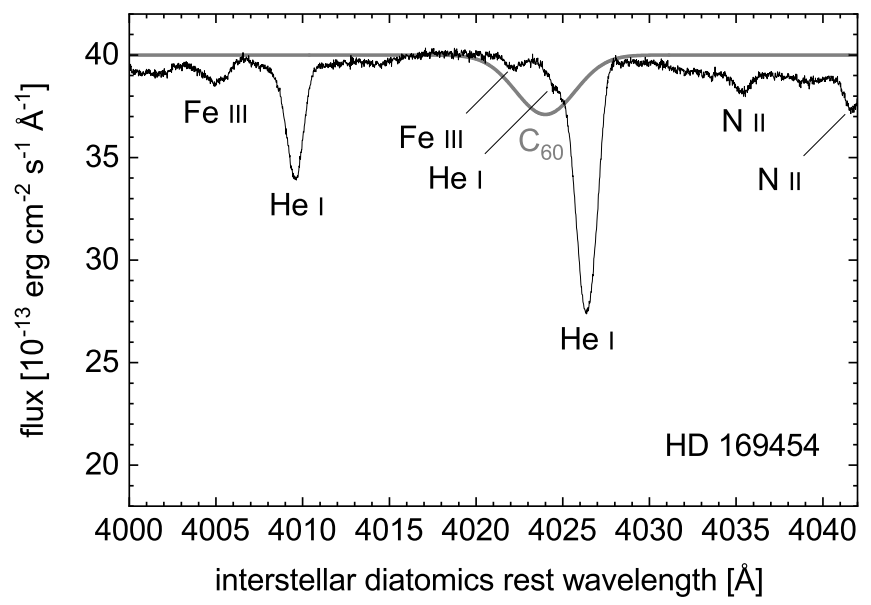

Fig. 2. Spectrum toward HD 169454 (black solid curve) and synthetic absorption spectrum of $\mathrm{C}_{60}$ with the expected band positions, widths, and relative areas (thick gray solid curve). Narrow absorption lines in the 3967-3971 $\AA$ interval correspond to velocity components of the Ca II $\mathrm{H}$ line.

line such as toward O9Ib-type HD 76341 may constitute a cause for concern.

\subsection{Observational data near $6000 \AA$}

Figure 4 shows the spectral region where the $\gamma_{0}$ band of $\mathrm{C}_{60}$ is expected. A straight line continuum was defined over the 6058$6087 \AA$ range of each original spectrum, still in the observer rest frame, and used to normalize the corresponding spectrum. The straight continuum was extrapolated to $6092 \AA$ so as to be applied to the $6090 \AA$ narrow DIB, the position of which was to serve as reference for evaluating those of other DIBs (Sect. 6). Next the wavelength scale was changed from observer rest frame to interstellar (Appendix B).

While the $4000 \AA$ spectral region is rich in stellar lines, the $6000 \AA$ region comprises several DIBs and, in most cases, a line of stellar Ne I measured at $6074.338 \AA$ in the laboratory (see Hobbs et al. 2009, for HD 183143). For all LOSs but HD 169454, the position of this line was predicted from the position of the He I line measured at 5875.62 $\AA$ in the laboratory. As to the spectrum toward HD 169454, we used lines of N II, Al III, and Si III that are observed clearly between 5665 and $5740 \AA$, for the He I line does not have a peak profile that would yield an accurate position.

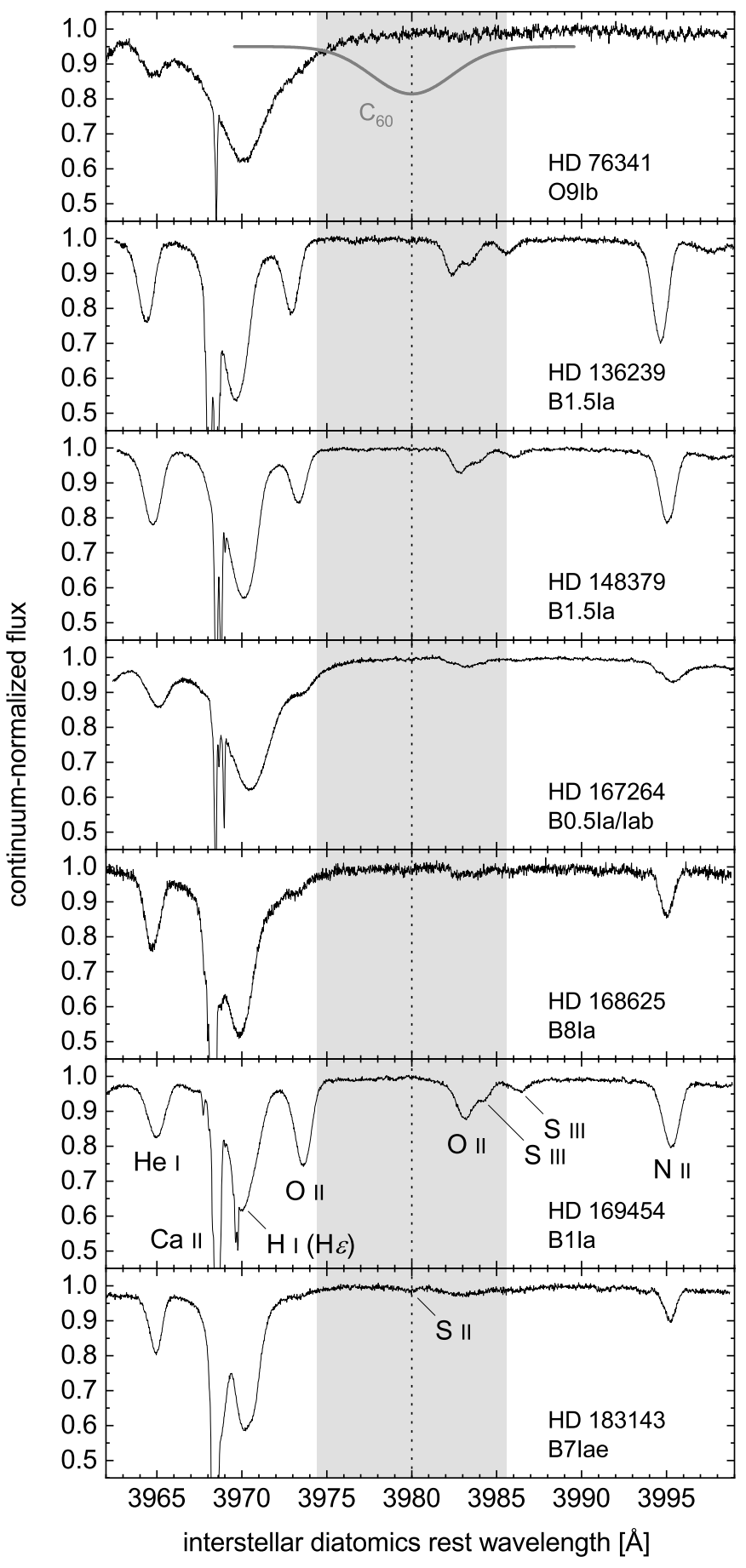

Fig. 3. Normalized observational spectra (black solid curves). A straight line continuum was defined over the 3960-4000 A range of each spectrum still in the observer rest frame. The top panel includes a synthetic $\mathrm{A}_{1}$ band of $\mathrm{C}_{60}$ with expected position and width, and arbitrary equivalent width (thick gray solid curve). Its position is indicated through all panels (vertical dotted line). The light gray area indicates the wavelength interval of two FWHMs taken into account to evaluate ${ }^{\mathrm{u}} W\left(\mathrm{C}_{60}\right.$, $\mathrm{A}_{1}$ ) at $3980 \AA$ for each LOS. The spectral type of each target star is indicated.

A shift is apparent between the DIBs in the spectrum toward HD 183143 and the positions reported by Hobbs et al. (2009) for this very LOS (Fig. 4). It is caused by a difference in the choice of reference for rest, the strongest velocity component of diatomic lines versus the strongest component of the $7698 \AA \mathrm{K}$ I 
line. The velocity difference is $\sim 15 \mathrm{~km} \mathrm{~s}^{-1}$ (Hobbs et al. 2009), representing $0.3 \AA$ at $6000 \AA$ as observed in Fig. 4 .

Finally, although $\mathrm{H}_{2} \mathrm{O}$ vapor gives numerous absorption lines near $6070 \AA$ (Carleer et al. 1999), they are very weak and generally not detected. Thus telluric features are not seen.

Two DIBs are found at 6068.4 and $6071.1 \AA$ (Galazutdinov et al. 2000; Tuairisg et al. 2000; Weselak et al. 2000; Hobbs et al. 2009), close to the position of the $\gamma_{0}$ band, that is, $6070 \pm 1 \AA$. Their FWHMs are on the order of $1-2 \AA$, as expected for the $\gamma_{0}$ band. There is, however, no further indication at this stage that either of these DIBs could be identified with $\gamma_{0}$. Although they represent rather weak absorptions, their presence influences the search for the $\mathrm{C}_{60}$ feature.

\section{Column density of $\mathrm{C}_{60}^{+}$}

The column density of $\mathrm{C}_{60}^{+}$can be determined by comparing the equivalent widths of the relevant DIBs with Gaussian profiles defined using the absorption cross sections and associated widths derived by Campbell et al. (2016b) for a temperature of $10 \mathrm{~K}$. We assume here that, for a given column density, $W$ is conserved over a broad range of conditions. Considering an absorption feature with a Gaussian profile in the wavelength domain, where $W$ is defined, the column density $N$ of the absorbing species derived from the Lambert-Beer law is such that

$N=-\frac{1}{\sigma} \ln \left[1-k \frac{W}{w}\right]$

Given the large uncertainties on $\sigma\left(\mathrm{C}_{60}^{+}, 9577.5 \AA\right)$ and $\sigma\left(\mathrm{C}_{60}^{+}\right.$, $9632.7 \AA)$, both $40 \%$, on $w\left(\mathrm{C}_{60}^{+}, 9577.5 \AA\right)$ and $w\left(\mathrm{C}_{60}^{+}, 9632.7 \AA\right)$, respectively 8 and $9 \%$, and on $W, 6-16 \%, \Delta N$ is computed by using the propagation formula for large errors proposed by Seiler (1987). We assume here that the errors are independent and correspond to normal distributions. A correction is applied accordingly to $N$ as calculated with Eq. (4) because the right hand term is a function that does not conserve the symmetry of normal distributions (Seiler 1987). The equivalent widths $W$ of the DIBs observed at $\sim 9577$ and $\sim 9632 \AA$, the column densities $N\left(\mathrm{C}_{60}^{+}\right)$derived from them, and the corresponding errors, are given in Table 1.

In several cases, $N\left(\mathrm{C}_{60}^{+}, 9577.5 \AA\right)$ and $N\left(\mathrm{C}_{60}^{+}, 9632.7 \AA\right)$ differ by a factor of $\sim 2$ although they are derived for the same LOS. Taking the uncertainties into account, the two values in each pair are nonetheless consistent. We note that the values presently obtained for $N\left(\mathrm{C}_{60}^{+}, 9577.5 \AA\right)$ toward HD 169454 and HD 183143, respectively $(7.8 \pm 4.7) \times 10^{12}$ and $(30 \pm 18) \times 10^{12} \mathrm{~cm}^{-2}$, are in agreement with those derived by Berné et al. (2017), respectively $9 \times 10^{12}$ and $20 \times 10^{12} \mathrm{~cm}^{-2}$ (Table C.1 in Berné et al. 2017).

\section{Column density of $\mathrm{C}_{60}$}

Bands of interstellar $\mathrm{C}_{60}$ have been looked for near $4000 \AA$ without success (e.g., Herbig 2000; Sassara et al. 2001; GarcíaHernández et al. 2012; García-Hernández \& Díaz-Luis 2013; Díaz-Luis et al. 2015). Similarly, the seven spectra in Fig. 3 do not show any clear indication of an absorption band around $3980.0 \AA$ that could be attributed to $\mathrm{C}_{60}$, and Fig. 4 does not reveal any new feature close to $6070.0 \AA$ either. As a consequence, column densities $N$ cannot be obtained for neutral

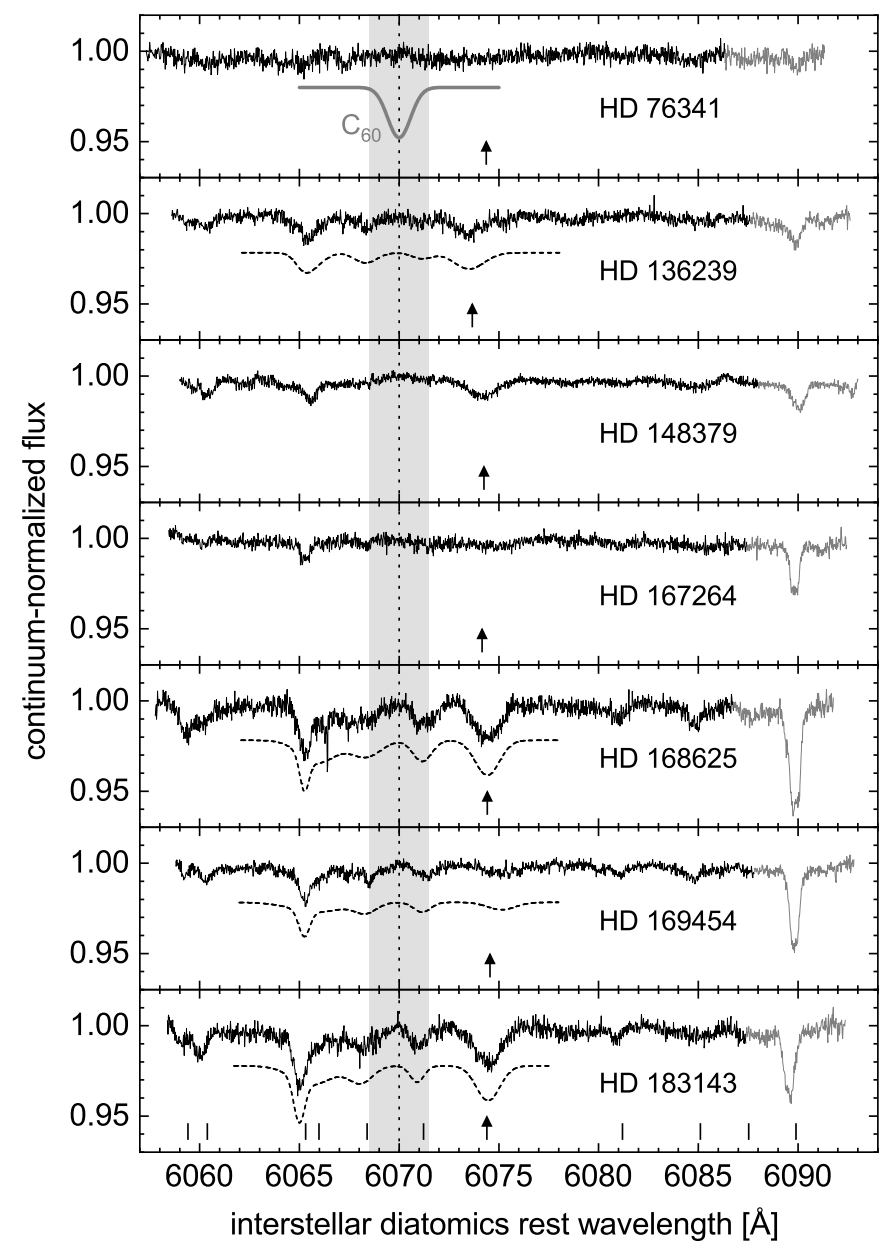

Fig. 4. Continuum-normalized spectra with interstellar wavelength scale for seven LOSs in the $6000 \AA$ region. Each gray portion was obtained by extrapolating the straight continuum defined over the 6058 $6087 \AA$ interval (observer rest frame). The top panel includes a synthetic $\gamma_{0}$ band of $\mathrm{C}_{60}$ of arbitrary equivalent width (thick gray solid curve, offset for clarity). Its position is indicated through all panels (vertical dotted line). Positions of DIBs according to Hobbs et al. (2009) are indicated in the bottom panel (short vertical lines). The predicted position of a line of stellar Ne I is marked in each panel (arrow). Synthetic spectra of DIBs and the Ne I line are represented in four panels (shortdashed curve). The light gray area indicates the wavelength interval of two FWHMs taken into account to evaluate ${ }^{u} W\left(\mathrm{C}_{60}, \gamma_{0}\right)$ at $6070 \AA$ for each LOS.

buckminsterfullerene. Nevertheless, upper limits denoted ${ }^{\mathrm{u}} \mathrm{N}$ can be estimated.

Values of ${ }^{\mathrm{u}} N$ can be evaluated by combining Eqs. (3) and (4). Observing that $k W$ is small compared to $w$, and replacing $N$ and $W$ by the corresponding upper limits ${ }^{\mathrm{u}} N$ and ${ }^{\mathrm{u}} W$, we obtain

${ }^{\mathrm{u}} N=\frac{4 \epsilon_{0} m c^{2}}{e^{2} \lambda^{2}} \frac{\mathrm{u}}{f}$,

or

${ }^{\mathrm{u}} N=1.1296 \times 10^{17} \frac{{ }^{\mathrm{u}} \mathrm{W}}{\lambda^{2} f}$,

with ${ }^{\mathrm{u}} N,{ }^{\mathrm{u}} W$, and $\lambda$ expressed in $\mathrm{cm}^{-2}, \mathrm{~m} \AA$, and $\AA$, respectively. One recognizes the formula used by Herbig (2000), Díaz-Luis et al. (2015), or Berné et al. (2017). We calculate $\Delta^{\mathrm{u}} N$ and rectify ${ }^{\mathrm{u}} N$ following Seiler (1987) to take the large error on $f$ into account. 
G. Rouillé et al.: The $\mathrm{C}_{60}: \mathrm{C}_{60}^{+}$ratio in diffuse and translucent interstellar clouds

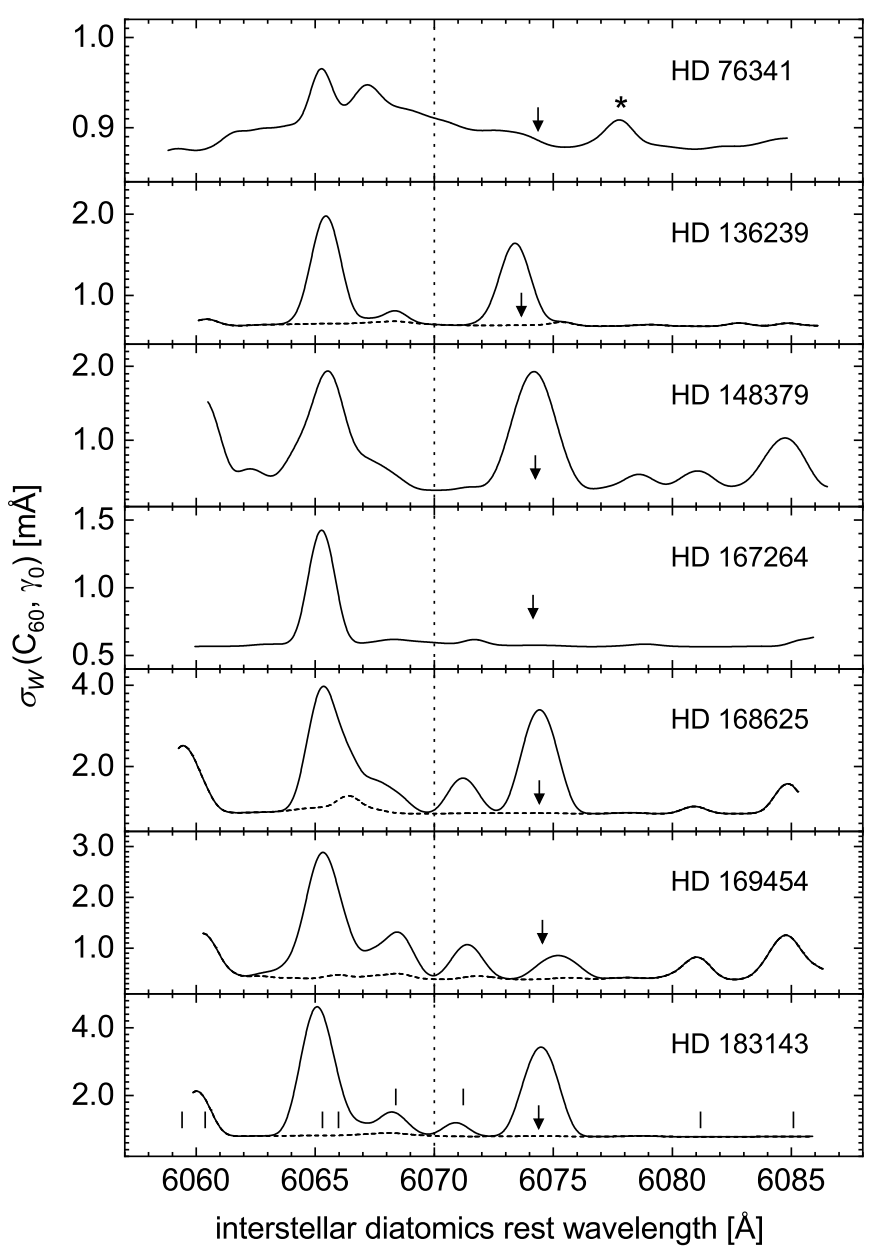

Fig. 5. Equivalent-width detection threshold in the $6000 \AA$ A region for seven LOSs (solid curves). The vertical dotted line shows the expected position of the $\gamma_{0}$ band of $\mathrm{C}_{60}$ in all spectra. Positions of DIBs according to Hobbs et al. (2009) are indicated in the bottom panel (short vertical lines). The predicted position of a line of stellar Ne I is marked in each panel (arrow). In four cases detection limits were computed as fitted profiles of the DIBs nearest $6070 \AA$ were included in the continuum (short-dashed curves). An unidentified absorption would cause the rise near $6078 \AA$ (asterisk).

Values of ${ }^{\mathrm{u}} W$, the upper limit for the equivalent width of the band expected at wavelength $\lambda$, are taken equal to three equivalent-width detection thresholds, that is, $3 \sigma_{W}$, where $\sigma_{W}$ is computed according to Lawton et al. (2008). Figure 5 presents $\sigma_{W}$ curves for the $6000 \AA$ region. Because $\sigma_{W}$ depends on the difference between the observed spectrum and the chosen continuum, $\sigma_{W}$ peaks at the position of any band or line that is not included in the continuum, as confirmed by a comparison with Fig. 4. Interestingly, the $\sigma_{W}$ curves reveal a possible absorption feature at a position varying from 6078 to $6079 \AA$, which we have not identified. The varying wavelength suggests it is not a DIB, though, unless it is the effect of a poorly defined shape.

Moreover, the value of $\sigma_{W}$ at a given wavelength considers the difference between the observed spectrum and the chosen continuum for all wavelengths in a surrounding interval spanning two FWHMs of the band to be detected. The value of $\sigma_{W}$ at this wavelength can then be lowered by including in the continuum any nearby band with a profile that extends into the interval. Figure 5 includes cases in which fitted profiles of the DIBs nearest $6070 \AA$ were included in the continuum. In the

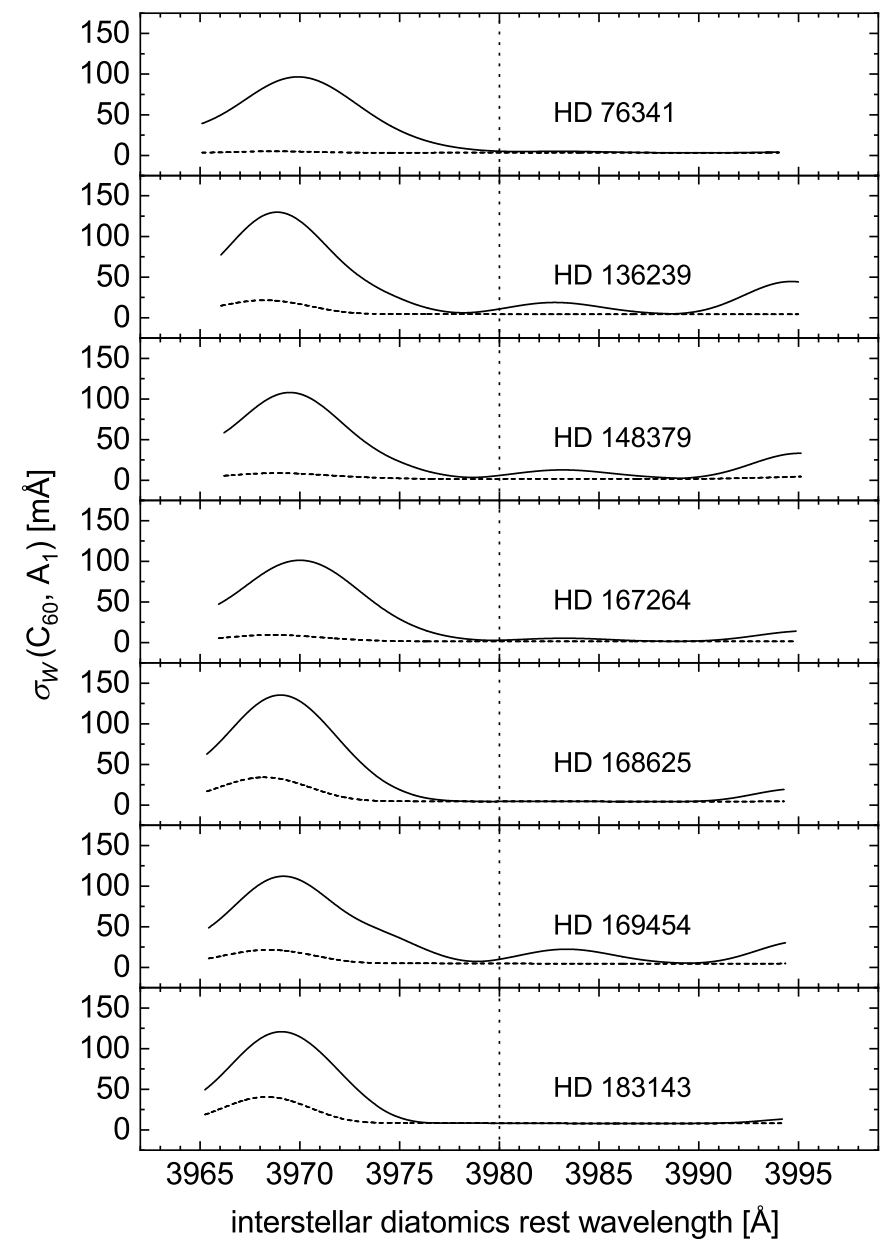

Fig. 6. Equivalent-width detection threshold in the $4000 \AA$ region scale for seven LOSs (solid curves). The vertical dotted line shows the expected position of the near-UV band of $\mathrm{C}_{60}$ in all spectra. The detection limits were also computed as fitted profiles of stellar lines were included in the continuum (short-dashed curves).

fitting procedure, each DIB was given a Gaussian profile with a position fixed to literature value (Hobbs et al. 2009) - corrected using the shift observed for the $6090 \AA$ DIB - while its FWHM and area were free. A poor fit results in an incomplete elimination of the feature contribution to the $\sigma_{W}$ curve.

Figure 6 shows $\sigma_{W}$ curves for the $4000 \AA$ region. They are given both with the stellar and interstellar atomic lines kept in the spectra and also with fitted profiles of the lines included in the continua. Large residuals from fitting the overlapping multiple components of the Ca II line cause the broad peak at $3968.5 \AA$ that appears after the procedure.

Table 2 presents values for ${ }^{\mathrm{u}} W\left(\mathrm{C}_{60}\right)$ and ${ }^{\mathrm{u}} N\left(\mathrm{C}_{60}\right)$ obtained from the normalized observational spectra shown in Figs. 3 and 4 . They were derived by giving $f\left(\mathrm{C}_{60}, \mathrm{~A}_{1}\right), w\left(\mathrm{C}_{60}, \mathrm{~A}_{1}\right)$, $f\left(\mathbf{C}_{60}, \gamma_{0}\right), w\left(\mathbf{C}_{60}, \gamma_{0}\right)$, and their uncertainties the values introduced and adopted in Sects. 3.2 and 3.4. The equivalent-width detection thresholds $\sigma_{W}$ yielding the ${ }^{\text {u }} W$ values were computed by using the data comprised within an interval centered at the rest wavelength of the relevant band and spanning two FWHMs.

According to the contents of Table 2, incorporation of specific spectral features into the continuum has the potential to lower substantially the upper limit for equivalent width. The most spectacular improvement is seen with HD 148379 where the ${ }^{\mathrm{u}} W$ and ${ }^{\mathrm{u}} N$ values derived from the UV spectrum are divided 
Table 2. Upper limits for column densities of interstellar $\mathrm{C}_{60}$ considering the $\mathrm{A}_{1}$ and $\gamma_{0}$ bands.

\begin{tabular}{|c|c|c|c|c|c|c|c|c|}
\hline \multirow[t]{2}{*}{ LOS } & \multicolumn{4}{|c|}{$\mathrm{C}_{60}, \mathrm{~A}_{1}(3980 \AA)$} & \multicolumn{4}{|c|}{$\mathrm{C}_{60}, \gamma_{0}(6070 \AA)$} \\
\hline & $\begin{array}{l}{ }^{\mathrm{u}} W \\
(\mathrm{~m} \AA)\end{array}$ & $\begin{array}{l}\Delta^{\mathrm{u}} W^{(a)} \\
(\mathrm{m} \AA)\end{array}$ & $\begin{array}{l}{ }^{\mathrm{u}} N \\
\left(10^{12} \mathrm{~cm}^{-2}\right)\end{array}$ & $\begin{array}{l}\Delta^{\mathrm{u}} N \\
\left(10^{12} \mathrm{~cm}^{-2}\right)\end{array}$ & $\begin{array}{l}{ }^{\mathrm{u}} W \\
(\mathrm{~m} \AA)\end{array}$ & $\begin{array}{l}\Delta^{\mathrm{u}} W^{(a)} \\
(\mathrm{m} \AA)\end{array}$ & $\begin{array}{l}{ }^{\mathrm{u}} N \\
\left(10^{12} \mathrm{~cm}^{-2}\right)\end{array}$ & $\begin{array}{l}\Delta^{\mathrm{u}} N \\
\left(10^{12} \mathrm{~cm}^{-2}\right)\end{array}$ \\
\hline \multirow[t]{2}{*}{ HD 76341} & 15.3 & 5.3 & 50 & 36 & 2.7 & 1.1 & 26 & 19 \\
\hline & 10.3 & 2.0 & 34 & 22 & $\ldots$ & $\ldots$ & $\ldots$ & $\ldots$ \\
\hline \multirow[t]{2}{*}{ HD 136239} & 32.1 & 7.9 & 106 & 70 & 1.93 & 0.79 & 18 & 13 \\
\hline & 13.1 & 2.3 & 43 & 27 & 1.93 & 0.79 & 18 & 13 \\
\hline \multirow[t]{2}{*}{ HD 148379} & 17.5 & 4.6 & 58 & 38 & 0.97 & 0.39 & 9.2 & 6.7 \\
\hline & 4.57 & 0.78 & 15.1 & 9.5 & $\ldots$ & $\ldots$ & $\ldots$ & $\ldots$ \\
\hline \multirow[t]{2}{*}{ HD 167264} & 8.4 & 2.8 & 28 & 19 & 1.78 & 0.73 & 17 & 12 \\
\hline & 4.25 & 0.75 & 14.0 & 8.9 & $\ldots$ & $\ldots$ & $\ldots$ & $\ldots$ \\
\hline \multirow[t]{2}{*}{ HD 168625} & 13.1 & 2.2 & 43 & 27 & 2.8 & 1.1 & 27 & 19 \\
\hline & 13.1 & 2.2 & 43 & 27 & 2.5 & 1.0 & 24 & 17 \\
\hline \multirow[t]{2}{*}{ HD 169454} & 29.6 & 6.8 & 98 & 64 & 1.37 & 0.59 & 13.0 & 9.7 \\
\hline & 14.1 & 2.5 & 46 & 29 & 1.16 & 0.46 & 11.0 & 8.0 \\
\hline \multirow[t]{2}{*}{ HD 183143} & 24.5 & 4.3 & 81 & 51 & 2.7 & 1.0 & 26 & 18 \\
\hline & 24.5 & 4.3 & 81 & 51 & 2.41 & 0.97 & 23 & 17 \\
\hline
\end{tabular}

Notes. Values in italic were obtained by including in the continuum the fitted profiles of atomic stellar lines when in the $4000 \AA$ A region, and those of the four DIBs nearest $6070 \AA$ and of the nearby Ne I line when in the $6000 \AA$ region. ${ }^{(a)}$ Uncertainty computed according to Lawton et al. (2008).

by a factor of close to four. The limits ${ }^{\mathrm{u}} N\left(\mathrm{C}_{60}, \mathrm{~A}_{1}\right)$ and ${ }^{\mathrm{u}} N\left(\mathrm{C}_{60}\right.$, $\gamma_{0}$ ) presented in Table 2 are in the ranges $14-81 \times 10^{12} \mathrm{~cm}^{-2}$ and $9-26 \times 10^{12} \mathrm{~cm}^{-2}$, respectively, when only the values obtained by including spectral features in the continuum, whenever available, are considered. The corresponding averages are $39 \times 10^{12} \mathrm{~cm}^{-2}$ and $18 \times 10^{12} \mathrm{~cm}^{-2}$. Thus ${ }^{\mathrm{u}} N\left(\mathrm{C}_{60}, \mathrm{~A}_{1}\right)$ is approximately twice as large as ${ }^{\mathrm{u}} N\left(\mathrm{C}_{60}, \gamma_{0}\right)$. The uncertainties are very large, with $63-65 \%$ for ${ }^{\mathrm{u}} N\left(\mathrm{C}_{60}, \mathrm{~A}_{1}\right)$ and $70-74 \%$ for ${ }^{\mathrm{u}} N\left(\mathrm{C}_{60}, \gamma_{0}\right)$.

\section{Upper limits for the $\mathrm{C}_{60}: \mathrm{C}_{60}^{+}$ratio}

In order to compare populations of $\mathrm{C}_{60}$ and $\mathrm{C}_{60}^{+}$in interstellar clouds, we assume that the angular size of the clouds before a target star is large in comparison with the difference in LOS coordinates during the observations at UV and visible wavelengths, which remains smaller than $5^{\prime \prime}$ (Table A.1). We also assume that the properties of the clouds do not vary significantly between the two sets of coordinates.

Our comparison is relevant to entire clouds and not to specific layers because the wavelengths of the DIBs attributed to $\mathrm{C}_{60}^{+}$ were determined by calibrating astronomical spectra with both atomic and molecular lines (Galazutdinov et al. 2017). The DIB wavelengths are given with an error in the range $0.1-0.3 \AA$, that is, on the order of the equivalent velocity difference between the diffuse atomic outer layer and the denser diatomic layer observed for HD 183143 (see Sect. 4.2). Moreover, the bands of $\mathrm{C}_{60}$ are currently not characterized with sufficient precision to allow us to search for them in a given layer.

The upper limit of the $\mathrm{C}_{60}: \mathrm{C}_{60}^{+}$ratio then equals ${ }^{\mathrm{u}} N\left(\mathrm{C}_{60}\right) / N\left(\mathrm{C}_{60}^{+}\right)$. It is calculated by combining Eqs. (4) and (6) into which the values given in Tables 1 and 2 are entered, and by adding a correction that large errors make necessary (Seiler 1987). The errors that affect the ratios are then obtained by taking into account those attached to the band descriptors rather than $\Delta^{\mathrm{u}} N$ and $\Delta N$. The results are given in Table 3 , showing that the upper limit of the $\mathrm{C}_{60}: \mathrm{C}_{60}^{+}$ratio noticeably depends on the band used to evaluate ${ }^{\mathrm{u}} N\left(\mathrm{C}_{60}\right)$. It ranges from 1.6 to 6.9 with an unweighted average value of 3.5 when using $\mathrm{A}_{1}$, and from 0.90 to 5.2 averaging 1.8 when using $\gamma_{0}$. While the values in the latter case are lower and less spread than in the former when the extreme value of 5.2 is excluded, their relative uncertainties are greater. In either case the uncertainties are very large, respectively $76-81$ and $83-90 \%$, owing essentially to poorly determined oscillator strengths and absorption cross sections. The average ratio of 1.8 obtained by using $\gamma_{0}$ is lowered to 1.3 when applying the inverted, squared errors as weights.

\section{Discussion}

\subsection{The $C_{60}: C_{60}^{+}$ratio in the diffuse ISM}

The detection of buckminsterfullerene and its cation in the ISM has extended the network of physical and chemical mechanisms at work in that environment. The relative populations of gasphase $\mathrm{C}_{60}, \mathrm{C}_{60}^{+}$, and $\mathrm{C}_{60}^{-}$would be primarily governed by the ISRF strength and the electron density, which are directly related to mechanisms such as photoionization of $\mathrm{C}_{60}$ and electron detachment from $\mathrm{C}_{60}^{-}$, electron attachment to $\mathrm{C}_{60}$ and electron recombination with $\mathrm{C}_{60}^{+}$.

Figure 7 illustrates ${ }^{\mathrm{u}} N\left(\mathrm{C}_{60}, \gamma_{0}\right) / N\left(\mathrm{C}_{60}^{+}\right)$as a function of $\mathrm{LOS}$ and includes literature values of $\mathrm{C}_{60}: \mathrm{C}_{60}^{+}$for comparison. The upper limits presently derived have an average value of 1.8 , lowered to 1.3 when applying the inverted, squared errors as weights. Both averages are compatible with the interval of ratio values 0.3-6 presented by Berné et al. (2017), in particular its lower half. They suggest, however, that the ratio of 5.6 proposed by Bakes \& Tielens (1995) in their theoretical study is somewhat large.

The value proposed by Bakes \& Tielens (1995) was determined for a typical diffuse cloud with $100 \mathrm{~K}$ gas temperature, $7.5 \times 10^{-3} \mathrm{~cm}^{-3}$ electron density, and $1 \mathrm{G}_{0}$ ISRF, conditions that may not be observed along the real LOSs presently studied. For instance, Gredel \& Münch (1986) determined a gas temperature of $15 \mathrm{~K}$, thus well below $100 \mathrm{~K}$, from the observation of $\mathrm{C}_{2}$ toward HD 169454. Additionally, in a study of $\mathrm{C}_{3}$ observed toward the same star, Schmidt et al. (2014) brought out two populations with excitation temperatures of $22.4 \pm 1 \mathrm{~K}$ and $187 \pm 25 \mathrm{~K}$. Their analysis of the colder or less excited population led to an ISRF with a strength of 2 to $6 \mathrm{G}_{0}$, greater than the $1 \mathrm{G}_{0}$ ISRF used by Bakes \& Tielens (1995). As to electron density, 
Table 3. Upper limits for $\mathrm{C}_{60}: \mathrm{C}_{60}^{+}$as ${ }^{\mathrm{u}} N\left(\mathrm{C}_{60}\right) / N\left(\mathrm{C}_{60}^{+}\right)$from the comparison of the $\mathrm{A}_{1}$ and $\gamma_{0}$ bands of $\mathrm{C}_{60}$ with the 9577.5 and $9632.7 \AA$ bands of $\mathrm{C}_{60}^{+}$.

\begin{tabular}{lllll}
\hline \hline \multirow{2}{*}{ LOS } & \multicolumn{2}{c}{$\mathrm{C}_{60}, \mathrm{~A}_{1}(3980 \AA)$} & \multicolumn{2}{c}{$\mathrm{C}_{60}, \gamma_{0}(6070 \AA)$} \\
& $\mathrm{C}_{60}^{+}, 9577.5 \AA$ & $\mathrm{C}_{60}^{+}, 9632.7 \AA$ & $\mathrm{C}_{60}^{+}, 9577.5 \AA$ & $\mathrm{C}_{60}^{+}, 9632.7 \AA$ \\
\hline HD 76341 & $6.2(5.3)$ & $4.1(3.6)$ & $3.1(2.8)$ & $2.1(1.9)$ \\
& $4.2(3.3)$ & $2.8(2.2)$ & $\ldots$ & $\ldots$ \\
HD 136239 & $8.2(6.5)$ & $9.1(7.4)$ & $1.4(1.2)$ & $1.6(1.4)$ \\
& $3.3(2.6)$ & $3.7(2.9)$ & $1.4(1.2)$ & $1.6(1.4)$ \\
HD 148379 & $7.3(5.9)$ & $7.4(6.0)$ & $1.2(1.0)$ & $1.2(1.0)$ \\
& $1.9(1.5)$ & $1.9(1.5)$ & $\ldots$ & $\ldots$ \\
HD 167264 & $8.5(7.5)$ & $4.1(3.5)$ & $5.2(4.7)$ & $2.5(2.2)$ \\
& $4.3(3.5)$ & $2.1(1.7)$ & $\ldots$ & $\ldots$ \\
HD 168625 & $2.1(1.6)$ & $1.6(1.3)$ & $1.3(1.1)$ & $1.01(0.88)$ \\
& $2.1(1.6)$ & $1.6(1.3)$ & $1.2(1.0)$ & $0.90(0.79)$ \\
HD 169454 & $14(12)$ & $8.6(6.9)$ & $1.9(1.7)$ & $1.1(1.0)$ \\
& $6.9(5.4)$ & $4.1(3.2)$ & $1.6(1.4)$ & $0.97(0.85)$ \\
HD 183143 & $4.0(3.1)$ & $6.3(5.1)$ & $1.3(1.1)$ & $2.0(1.8)$ \\
& $4.0(3.1)$ & $6.3(5.1)$ & $1.12(0.97)$ & $1.8(1.6)$ \\
\hline
\end{tabular}

Notes. Values between parentheses are computed errors. Values in italic were obtained by including in the continuum the fitted profiles of atomic stellar lines when in the $4000 \AA$ region, and those of the four DIBs nearest $6070 \AA$ and of the nearby Ne I line when in the $6000 \AA$ region.

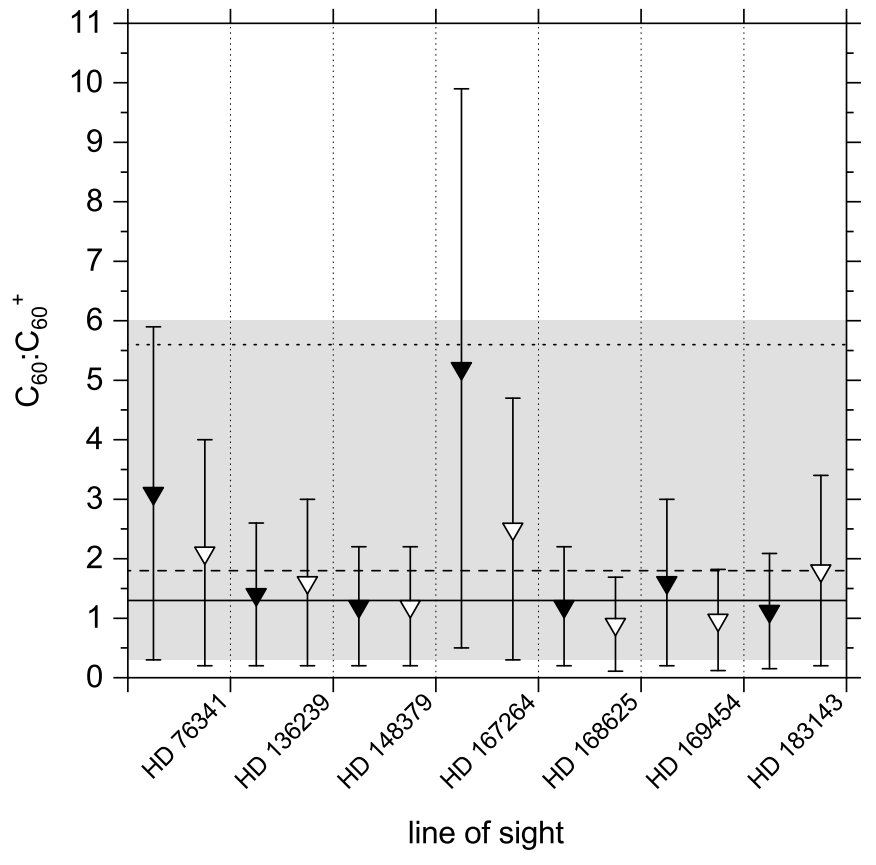

Fig. 7. Values of $\mathrm{C}_{60}: \mathrm{C}_{60}^{+}$in the diffuse ISM as a function of LOS (see Table 3). Triangle symbols $(\boldsymbol{\nabla}$ and $\nabla)$ : upper limits ${ }^{\mathrm{u}} N\left(\mathrm{C}_{60}, \gamma_{0}\right) / N\left(\mathrm{C}_{60}^{+}\right.$, $\lambda$ ) with $\lambda=9577.5 \AA$ and $\lambda=9632.7 \AA$, respectively. Error bars correspond to errors given in Table 3. Gray area: $\mathrm{C}_{60}: \mathrm{C}_{60}^{+}$according to Berné et al. (2017). Horizontal dotted line: $\mathrm{C}_{60}: \mathrm{C}_{60}^{+}$for a typical diffuse medium as derived by Bakes \& Tielens (1995). Horizontal dashed line: mean value of the present upper limits. Horizontal solid line: weighted mean value of the present upper limits. Vertical dotted lines separate the data relevant to each LOS.

Harrison et al. (2013) proposed typical values in the range 0.01$0.06 \mathrm{~cm}^{-3}$, up to eight times that adopted by Bakes \& Tielens (1995). Thus the conditions assumed by Bakes \& Tielens (1995) may not be adequate to describe our LOSs, resulting through their interplay in a $\mathrm{C}_{60}: \mathrm{C}_{60}^{+}$value greater than the weighted average upper limit derived in this study.
Moreover, properties of $\mathrm{C}_{60}$ and its ions relevant to the determination of their relative populations are not accurately known. The $\mathrm{C}_{60}^{-}: \mathrm{C}_{60}$ and $\mathrm{C}_{60}: \mathrm{C}_{60}^{+}$ratios are proportional, respectively, to the electron attachment rate of $\mathrm{C}_{60}$ and the electron recombination rate of $\mathrm{C}_{60}^{+}$. To date, both values are uncertain. Bakes \& Tielens (1995) attributed to neutral $\mathrm{C}_{60}$ an electron attachment cross section of $10^{-12} \mathrm{~cm}^{2}$ in their typical diffuse medium, a value proposed by Lezius et al. (1993). Because the analysis of measurements for electrons with low energy is complex (Kasperovich et al. 2001; Lezius 2003), the actual attachment cross section for an electron with an energy of $\sim 0.013 \mathrm{eV}$ (corresponding to $100 \mathrm{~K}$ ) may be different, and, consequently, $\mathrm{C}_{60}^{-}: \mathrm{C}_{60}$ too. As to the electron recombination rate of $\mathrm{C}_{60}^{+}$, which has yet to be measured, Bakes \& Tielens (1995) used a theoretical value extrapolated from a model for grains (Draine \& Sutin 1987). Yet they noted that this model gave rates an order of magnitude larger than measured ones when it was applied to the benzene $\left(\mathrm{C}_{6} \mathrm{H}_{6}\right)$ and naphthalene $\left(\mathrm{C}_{10} \mathrm{H}_{8}\right)$ cations. Thus, the value they obtained for $\mathrm{C}_{60}: \mathrm{C}_{60}^{+}$is possibly overestimated.

As to our result, one must be aware of the large uncertainties affecting the absorption cross sections and oscillator strengths we employed to evaluate $\mathrm{C}_{60}: \mathrm{C}_{60}^{+}$. Any correction applied to one of them changes ${ }^{\mathrm{u}} N\left(\mathrm{C}_{60}\right) / N\left(\mathrm{C}_{60}^{+}\right)$, and therefore $\mathrm{C}_{60}: \mathrm{C}_{60}^{+}$, in proportion.

The influence of the chosen oscillator strengths on the $\mathrm{C}_{60}: \mathrm{C}_{60}^{+}$ratio is illustrated by the following evaluations, which differ from ours. Fulara et al. (1993) had estimated for the two bands of $\mathrm{C}_{60}^{+}$a total oscillator strength in the range 0.003-0.006, that is, five to nine times smaller than the value of 0.0278 we adopted (from adding 0.0164 to 0.0114 , see Sect. 3.3). The former value was used by Herbig (2000) to estimate $N\left(\mathrm{C}_{60}^{+}\right)$toward Cyg OB2/8A, which, combined with an evaluation of ${ }^{\mathrm{u}} N\left(\mathrm{C}_{60}\right)$ lower than ours by an order of magnitude, led to $\mathrm{C}_{60}: \mathrm{C}_{60}^{+}$being 0.0075 at most. In that study, the author mentioned his doubts concerning the result and explained how the value for ${ }^{\mathrm{u}} N\left(\mathrm{C}_{60}\right)$ might be incorrect owing to a possibly much underestimated FWHM (see Sect. 8.2). In a second case, Maier \& Campbell (Maier \& Campbell 2017; Campbell \& Maier 2017) inevitably derived a high degree of ionization, $98 \%$, because they borrowed 
${ }^{\mathrm{u}} N\left(\mathrm{C}_{60}\right)$ from Herbig (2000). They also stated that the degree of ionization should be at least $90 \%$ even if one of the DIBs observed near $6000 \AA$ was absorption by $\mathrm{C}_{60}$ (Maier \& Campbell 2017). The value was derived from elements in a discussion by Omont (2016), among which a theoretical oscillator strength of 0.01 attributed to the potential $\mathrm{C}_{60}$ DIB, which is 25 times greater than the strength presently determined for the $\gamma_{0}$ band. We remark that abundances of neutral molecules and cations as evaluated by Omont (2016) would give $80 \%$ as the degree of ionization.

Finally, when using Eqs. (4) and (5), we assume that the area of the band is a valid measurement of the population of the absorbing species in absence of vibrational excitation, and that it is conserved over the range of temperature observed in diffuse and translucent clouds. Taking into account the conditions of the laboratory measurements on $\mathrm{C}_{60}$ and $\mathrm{C}_{60}^{+}$, the bands that interest us represent transitions from the electronic ground state with zero quantum of vibrational excitation. As a transfer of population to excited vibrational levels occurs when the temperature increases, the validity of the computed column densities may be affected when looking at the warmer regions of diffuse clouds. Nevertheless, provided the vibrational modes of the initial and final states of a transition are similar, the loss of area caused by an increase in the temperature may be compensated by the rise of overlapping bands that correspond to transitions from the thermally populated vibrational levels with conservation of the vibrational state. In this case, the $\mathrm{C}_{60}: \mathrm{C}_{60}^{+}$ratio may be valid for the range of temperatures encountered throughout molecular clouds. Verification is needed.

As mentioned in the introduction, Iglesias-Groth (2019) observed interstellar fullerenes $\mathrm{C}_{60}$ and $\mathrm{C}_{70}$, and the ions $\mathrm{C}_{60}^{+}$and $\mathrm{C}_{60}^{-}$, in IR emission spectra of the star-forming region IC 348 . Ion fractions of 20 and $10 \%$ were determined for $\mathrm{C}_{60}^{+}$and $\mathrm{C}_{60}^{-}$, respectively, giving a $\mathrm{C}_{60}: \mathrm{C}_{60}^{+}$ratio of 3.5. We do not attempt a comparison with our results because the ISRF in the targeted regions is probably much stronger than in the typical diffuse or translucent interstellar clouds we are interested in. Actually Iglesias-Groth (2019) adopted ISRF strengths of 20 and $45 \mathrm{G}_{0}$ in their analysis. Moreover, we are not aware of an evaluation of the electron density in the relevant regions. This value would have to be taken into account (Bakes \& Tielens 1994, 1995).

\subsection{Searches for vibronic bands of $C_{60}$ in the diffuse ISM}

Searches for interstellar $\mathrm{C}_{60}$ were attempted following the report of a narrow absorption by $\mathrm{C}_{60}$ at $3860 \AA$ (Snow \& Seab 1989; Somerville \& Bellis 1989; Somerville \& Crawford 1993). The measurements concerned van der Waals complexes of $\mathrm{C}_{60}$ with benzene and dichloromethane, from which the band of free $\mathrm{C}_{60}$ was predicted and attributed to the origin of the $1^{1} \mathrm{~T}_{1 u} \leftarrow{ }^{1} \mathrm{~A}_{g}$ transition (Heath et al. 1987). Because it is firmly established that this transition is found at $4024 \AA$ as the $\mathrm{A}_{0}$ band, the upper limit, on the order of $10^{14} \mathrm{~cm}^{-2}$, derived by Snow \& Seab (1989) for $N\left(\mathrm{C}_{60}\right)$ required a revision that Herbig (2000) attempted, though without obtaining a satisfying result.

Ehrenfreund \& Foing (1997) searched for absorption by $\mathrm{C}_{60}$ near 3980 and near $6070 \AA$ in a spectrum measured toward $\mathrm{BD}+63^{\circ} 1964$. They found a weak band with $20 \mathrm{~m} \AA$ equivalent width close to $3980 \AA$. At $6070 \AA$, with a detection limit of $3 \mathrm{~m} \AA$ equivalent width, the spectrum did not show any absorption band. A possible DIB observed at $6220 \AA$ appeared to coincide with a band seen beside the $\gamma_{0}$ band in the spectrum of $\mathrm{C}_{60}$ (Haufler et al. 1991). Because laboratory spectra indicate that the $\gamma_{0}$ band is the strongest component in the pair, the DIB at $6220 \AA$ is likely caused by an interstellar substance that is not $\mathrm{C}_{60}$. Additionally, the wavelengths of the bands observed by Ehrenfreund \& Foing (1997) do not coincide exactly with those derived from laboratory spectra of $\mathrm{C}_{60}$ (Herbig 2000).

Herbig (2000) looked for various bands of $\mathrm{C}_{60}$ toward several objects. Using band C reported by Leach et al. (1992) at $3284 \AA$ in hexane at $300 \mathrm{~K}$, an upper limit of $4.5 \times 10^{11} \mathrm{~cm}^{-2}$ was found for $N\left(\mathrm{C}_{60}\right)$ toward Cyb OB2/8A. The value is invalid, as actually suspected by the author, because it was derived by hypothesizing an FWHM of $1 \AA$ for band $\mathrm{C}$ in the gas phase, which is too low a value considering that the band has a width of $1660 \mathrm{~cm}^{-1}$ in the spectrum of $\mathrm{C}_{60}$ in Ne ice (Sassara et al. 2001), that is, $173 \AA$ (see also Fig. 1). Using this underestimated FWHM to evaluate ${ }^{\mathrm{u}} N\left(\mathrm{C}_{60}, \mathrm{C}\right)$, Herbig (2000) obtained a $\mathrm{C}_{60}: \mathrm{C}_{60}^{+}$ratio on the order of 0.01 at maximum (see Sect. 8.1).

García-Hernández et al. (2012), García-Hernández \& Díaz-Luis (2013), and Díaz-Luis et al. (2015) sought $\mathrm{C}_{60}$ toward the hot star DY Cen and the planetary nebulae Tc 1 and IC 418. From $1 \sigma$ equivalent-width detection limits at $3980 \AA$, the authors derived upper limits of $1.5 \times 10^{13}, 1 \times 10^{13}$, and $4 \times 10^{13} \mathrm{~cm}^{-2}$ for the column densities of $\mathrm{C}_{60}$ toward DY Cen, Tc 1, and IC 418 , respectively. The ${ }^{\mathrm{u}} N\left(\mathrm{C}_{60}, \mathrm{~A}_{1}\right)$ values we obtained are on the same order (Table 2), though we took into account three equivalent-width detection thresholds in the form of ${ }^{u} W$. Analysis using the $\gamma_{0}$ band gave lower values, albeit on the same order, despite again taking three equivalent-width detection thresholds into account.

Past searches for interstellar $\mathrm{C}_{60}$ focused on the detection of its UV and near-UV bands. To our knowledge, only Ehrenfreund \& Foing (1997) and Herbig (2000) examined the possibility that DIBs near $6070 \AA$ could be related to $\mathrm{C}_{60}$. Because it is narrow and lies at a visible wavelength, the $\gamma_{0}$ band is a more convenient target than, for instance, $\mathrm{A}_{1}$, as demonstrated by the ${ }^{\mathrm{u}} \mathrm{W}$ values in Table 2, despite the larger uncertainties that affect the description of this absorption. Furthermore, spectra in rare-gas matrices showed that the $\gamma_{2}$ band is similar both in strength and width to $\gamma_{0}$ (Gasyna et al. 1991; Sassara et al. 1997, and this work). Solvatochromism measurements (Catálan 1994; Renge 1995) and low-temperature gas-phase spectroscopy (Hansen et al. 1997) placed $\gamma_{2}$ at $\sim 584 \mathrm{~nm}$. It is desirable that a future search for $\mathrm{C}_{60}$ targets both bands. Already surveys list DIBs at positions close to those of the $\gamma_{0}$ and $\gamma_{2}$ bands. A comparison is not straightforward, however, because of the uncertainties that affect the laboratory data (see also Herbig 2000). Our simple analysis of DIB measurements toward three targets (Tuairisg et al. 2000) indicates that the most strongly correlated DIBs near the positions of $\gamma_{0}$ and $\gamma_{2}$ are the bands at 5828.56 and $6068.45 \AA$. Not taking uncertainties into account, the separation is $10 \AA$ too large for assigning the pair to the $\gamma_{0}$ and $\gamma_{2}$ bands.

\section{Conclusions}

After reevaluating the oscillator strengths of bands in the absorption spectrum of $\mathrm{C}_{60}$, we exploited spectra of background stars and available DIB measurements to estimate the $\mathrm{C}_{60}: \mathrm{C}_{60}^{+}$ratio in diffuse and translucent clouds. We obtained an average upper limit of $\sim 1.3$, corresponding to a minimum degree of ionization of $\sim 40 \%$. Combining observed emission and absorption bands of fullerene $\mathrm{C}_{60}$ and its cation, respectively, Berné et al. (2017) determined a $\mathrm{C}_{60}: \mathrm{C}_{60}^{+}$ratio equal to $0.3-6$ in the diffuse ISM. The two results present common values, and they suggest that the fraction of neutral molecules in the fullerene population of the 
G. Rouillé et al.: The $\mathrm{C}_{60}: \mathrm{C}_{60}^{+}$ratio in diffuse and translucent interstellar clouds

diffuse ISM may be notable even though its electronic transitions have not been detected. Our approach allows us, however, to not rely on IR emission spectra, which present a degree of ambiguity as to the species that cause them. As to the difference between our result and the theoretical prediction by Bakes \& Tielens (1995), it can simply be the consequence of inadequate values given to parameters of the model. In that respect, more accurate measurements of interstellar absorption at near-UV and visible wavelengths would be useful to constrain the local ISRF strength and electron density. Furthermore, the comparison of these measurements with those of mid-IR emission bands would allow us to distinguish the part of emission in diffuse and translucent clouds that is actually caused by gas-phase $\mathrm{C}_{60}$ molecules.

The very large uncertainties of the upper limits we derived are caused by the lack of accurate experimental data. New experiments are needed in order to better determine the absorption cross sections, oscillator strengths, and FWHMs of the various bands involved in this study, in particular that of the $\gamma_{0}$ band of $\mathrm{C}_{60}$ at $6070 \AA$. Because it is narrow and arises in a wavelength domain favorable to observations, the $\gamma_{0}$ band may be used to obtain the lowest equivalent-width detection threshold values. A better characterization of the $\gamma_{2}$ band is also desirable. Since matrix-isolation measurements showed its strength and width are similar to those of $\gamma_{0}$, a search for interstellar $\mathrm{C}_{60}$ at visible wavelengths would be considered successful if it revealed both bands. Another way to confirm the detection of $\gamma_{0}$ would be to observe the near-UV $A_{1}$ band too, which is especially worth attempting when the spectral type of the target star is favorable.

Finally, we need electronic spectra of $\mathrm{C}_{60}^{-}$at low temperature in the gas phase in order to progress. To date, measurements of vibronic bands of the anion are scarce and their resolution modest (Tomita et al. 2005; Støchkel \& Andersen 2013).

Acknowledgements. The authors gratefully acknowledge the use of observations collected at the European Southern Observatory under ESO programs 079.C0597 (R. Gredel, for HD 169454 and HD 183143), 081.C-0475 (J. Smoker, for HD 76341, HD 136239, and HD 167264), 082.C-0566 (Y. Beletsky, for HD 136239 and HD 148379), 194.C-0833 (N. Cox, for HD 167264, HD 169454, and HD 183143), and 266.D-5655 (Paranal Observatory, ESO, for HD 168625) S.K. is thankful to the Deutsche Forschungsgemeinschaft (DFG) for its support through project No. 413610339. Special thanks are owed to R. Gredel for discussions on the wavelength calibration of astronomical spectra and for suggesting analysis methods, and to D. Strelnikov for exchanges on the determination of oscillator strengths.

\section{References}

Bakes, E. L. O., \& Tielens, A. G. G. M. 1994, ApJ, 427, 822

Bakes, E. L. O., \& Tielens, A. G. G. M. 1995, Astrophys. Space Sci. Lib., 202, 315

Berné, O., Cox, N. L. J., Mulas, G., \& Joblin, C. 2017, A\&A, 605, L1

Brieva, A. C., Gredel, R., Jäger, C., Huisken, F., \& Henning, T. 2016, ApJ, 826, 122

Cami, J. 2014, in Proceedings of the International Astronomical Union, The Diffuse Interstellar Bands, eds. J. Cami, \& N. Cox (Cambridge: Cambridge University Press ), 9, 370

Cami, J., Bernard-Salas, J., Peeters, E., \& Malek, S. E. 2010, Science, 329, 1180

Campbell, E. K., \& Maier, J. P. 2017, J. Chem. Phys., 146, 160901

Campbell, E. K., Holz, M., Gerlich, D., \& Maier, J. P. 2015, Nature, 523, 322

Campbell, E. K., Holz, M., \& Maier, J. P. 2016a, ApJ, 826, L4

Campbell, E. K., Holz, M., Maier, J. P., et al. 2016b, ApJ, 822, 17

Cardelli, J. A., Clayton, G. C., \& Mathis, J. S. 1989, ApJ, 345, 245

Carleer, M., Jenouvrier, A., Vandaele, A.-C., et al. 1999, J. Chem. Phys., 111, 2444

Catálan, J. 1994, Chem. Phys. Lett., 223, 159

Catálan, J., \& Pérez, P. 2002, FNCN, 10, 171

Chupka, W. A., \& Klots, C. E. 1997, IJMSI, 167/168, 595

Close, J. D., Federmann, F., Hoffmann, K., \& Quaas, N. 1997, Chem. Phys Lett., 276, 393
Cordiner, M. A., Linnartz, H., Cox, N. L. J., et al. 2019, ApJ, 875, L28

Díaz-Luis, J. J., García-Hernández, D. A., Rao, N. K., Manchado, A., \& Cataldo, F. 2015, A\&A, 573, A97

Draine, B. T., \& Sutin, B. 1987, ApJ, 320, 803

Edwards, S. A., \& Leach, S. 1993, A\&A, 272, 533

Ehrenfreund, P., \& Foing, B. H. 1997, AdSpR, 19, 1033

Fan, H., Hobbs, L. M., Dahlstrom, J. A., et al. 2019, ApJ, 878, 151

Foing, B. H., \& Ehrenfreund, P. 1994, Nature, 369, 296

Foing, B. H., \& Ehrenfreund, P. 1997, A\&A, 319, L59

Fulara, J., Jakobi, M., \& Maier, J. P. 1993, Chem. Phys. Lett., 211, 227

Galazutdinov, G. A., Musaev, F. A., Krełowski, J., \& Walker, G. A. H. 2000 PASP, 112, 648

Galazutdinov, G., Krełowski, J., Beletsky, Y., \& Valyavin, G. 2015, PASP, 127, 356

Galazutdinov, G. A., Shimansky, V. V., Bondar, A., Valyavin, G., \& Krełowski, J. 2017, MNRAS, 465, 3956

García-Hernández, D. A., \& Díaz-Luis, J. J. 2013, A\&A, 550, L6

García-Hernández, D. A., Rao, N. K., \& Lambert, D. L. 2012, ApJ, 759, L21

García-Hernández, D. A., Cataldo, F., \& Manchado, A. 2013, MNRAS, 434, 415

Gasyna, Z., Schatz, P. N., Hare, J. P., et al. 1991, Chem. Phys. Lett., 183, 283

Gredel, R., \& Münch, G. 1986, A\&A, 154, 336

Hansen, K., Müller, R., Brockhaus, P., Campbell, E. E. B., \& Hertel, I. V. 1997, Z. Phys. D, 42, 153

Harrison, S., Faure, A., \& Tennyson, J. 2013, MNRAS, 435, 3541

Haufler, R. E., Chai, Y., Chibante, L. P. F., et al. 1991, J. Chem. Phys., 95, 2197

Heath, J. R., Curl, R. F., \& Smalley, R. E. 1987, J. Chem. Phys., 87, 4236

Herbig, G. H. 2000, ApJ, 542, 334

Hobbs, L. M., York, D. G., Thorburn, J. A., et al. 2009, ApJ, 705, 32

Hora, J., Pánek, P., Navrátil, K., et al. 1996, Phys. Rev. B, 54, 5106

Hunter, I., Smoker, J. V., Keenan, F. P., et al. 2006, MNRAS, 367, 1478

Iglesias-Groth, S. 2019, MNRAS, 489, 1509

Jannuzi, B. T., Black, J. H., Lada, C. J., \& van Dishoeck, E. F. 1988, ApJ, 332, 995

Kasperovich, V., Tikhonov, G., \& Kresin, V. V. 2001, Chem. Phys. Lett., 337, 55

Krasnokutski, S. A., Gruenewald, M., Jäger, C., et al. 2019, ApJ, 874, 149

Lawton, B., Churchill, C. W., York, B. A., et al. 2008, AJ, 136, 994

Leach, S., Vervloet, M., Desprès, A., et al. 1992, Chem. Phys., 160, 451

Léger, A., d'Hendecourt, L., Verstraete, L., \& Schmidt, W. 1988, A\&A, 203, 145

Lezius, M. 2003, IJMSp, 223-224, 447

Lezius, M., Scheier, P., \& Märk, T. D. 1993, Chem. Phys. Lett., 203, 232

Maier, J. P., \& Campbell, E. K. 2017, Angew. Chem. Int. Ed., 56, 4920

Mulliken, R. S. 1939, J. Chem. Phys., 7, 14

Negri, F., Orlandi, G., \& Zerbetto, F. 1992, J. Chem. Phys., 97, 6496

Omont, A. 2016, A\&A, 590, A52

Orlandi, G., \& Negri, F. 2002, Photochem. Photobiol. Sci., 1, 289

Pan, K., Federman, S. R., Sheffer, Y., \& Andersson, B.-G. 2005, ApJ, 633, 986

Renge, I. 1995, J. Phys. Chem., 99, 15955

Rice, J. S., Federman, S. R., Flagey, N., et al. 2018, ApJ, 858, 111

Rouillé, G., Steglich, M., Huisken, F., Henning, T., \& Müllen, K. 2009, J. Chem. Phys., 131, 204311

Sassara, A., Zerza, G., Chergui, M., Negri, F., \& Orlandi, G. 1997, J. Chem. Phys., 107, 8731

Sassara, A., Zerza, G., Chergui, M., \& Leach, S. 2001, ApJS, 135, 263

Schmidt, M. R., Krełowski, J., Galazutdinov, G. A., et al. 2014, MNRAS, 441, 1134

Seiler, F. A. 1987, Risk Anal., 7, 509

Sellgren, K., Werner, M. W., Ingalls, J. G., et al. 2010, ApJ, 722, L54

Snow, T. P., \& McCall, B. J. 2006, ARA\&A, 44, 367

Snow, T. P., \& Seab, C. G. 1989, A\&A, 213, 291

Somerville, W. B., \& Bellis, J. G. 1989, MNRAS, 240, 41

Somerville, W. B., \& Crawford, I. A. 1993, Faraday Trans., 89, 2261

Sowers, B. L., Williams, M. W., Hamm, R. N., \& Arakawa, E. T. 1972, J. Chem. Phys., 57, 167

Støchkel, K., \& Andersen, J. U. 2013, J. Chem. Phys., 139, 164304

Strelnikov, D., Kern, B., \& Kappes, M. M. 2015, A\&A, 584, A55

Thompson, H. M. A., Keenan, F. P., Dufton, P. L., et al. 2008, MNRAS, 383, 729

Tomita, S., Andersen, J. U., Bonderup, E., et al. 2005, Phys. Rev. Lett., 94, 053002

Tuairisg, S. Ó., Cami, J., Foing, B. H., Sonnentrucker, P., \& Ehrenfreund, P. 2000, A\&AS, 142, 225

Walker, G. A. H., Bohlender, D. A., Maier, J. P., \& Campbell, E. K. 2015, ApJ, 812, L8

Walker, G. A. H., Campbell, E. K., Maier, J. P., Bohlender, D., \& Malo, L. 2016, ApJ, 831, 130

Walker, G. A. H., Campbell, E. K., Maier, J. P., \& Bohlender, D. 2017, ApJ, 843, 56

Weselak, T., Schmidt, M., \& Krełowski, J. 2000, A\&AS, 142, 239 


\section{Appendix A: Observational data}

For each LOS and wavelength domain examined in the present study, Table A.1 gives the resolution of the observational spectra, the angular distance to the target star, and the data sets. Information on the LOSs such as the spectral type of the target star and the observed color excess can be found for instance in Hunter et al. (2006) for HD 76341, HD 136239, HD 148379, HD 167264, and HD 169454, and in Fan et al. (2019) for HD 168625 and HD 183143. Selected information on each target star is also found in Galazutdinov et al. (2017).

\section{Appendix B: Wavelengths and standards of rest}

In this work, wavelengths are expressed in units of angstrom $(\AA)$ when explicit information shows that they were measured in air. Wavelengths are given in units of nanometer (nm) whenever the calibration conditions are not indicated in the source material. Wavenumbers in reciprocal centimeter $\left(\mathrm{cm}^{-1}\right)$ may be for air or vacuum.

For each LOS we examined lines of identified interstellar species to evaluate their radial velocity relative to the observer, that is, the wavelength standard of rest in the archival spectra. This information was used to change this standard for an interstellar absorber plausibly coexisting with fullerenes, allowing us to locate bands of interstellar $\mathrm{C}_{60}$ in a straightforward manner by using the laboratory rest wavelengths.

We are looking for interstellar $\mathrm{C}_{60}$ in diffuse and translucent molecular clouds, for which $\mathrm{K} \mathrm{I}, \mathrm{CH}$, and $\mathrm{CH}^{+}$are the preferred tracers of the less dense regions, and $\mathrm{CN}$ of the denser ones (Pan et al. 2005; Rice et al. 2018). In some cases, DIBs exhibit a shift relative to the lines of these tracers, indicating that cloud structures may be complex (Galazutdinov et al. 2015). Red and blue shifts as great as $\sim 10 \mathrm{~km} \mathrm{~s}^{-1}$ in terms of radial velocity have been observed. Because bands of interstellar $\mathrm{C}_{60}$ may be affected similarly, the $\gamma_{0}$ and $\mathrm{A}_{1}$ bands may be shifted respectively up to $0.2 \AA$ and $0.13 \AA$ toward shorter or longer wavelengths. This is not a major issue considering the respective FWHMs of $1.5 \pm$ $0.5 \AA$ and $5.54 \pm 0.80 \AA$ that the two bands may show (Sect. 3.2).

The near-UV and violet regions show several lines of interstellar atoms and molecules that can serve as references, among which $\mathrm{KI}, \mathrm{CN}, \mathrm{CH}$, and $\mathrm{CH}^{+}$are found (see, e.g., Hobbs et al. 2009; Galazutdinov et al. 2015). The K I lines, at 4044.136 and $4047.208 \AA$ rest wavelengths, are too weak to be seen in most of the present spectra though. In several cases, the $\mathrm{CN}, \mathrm{CH}$, and $\mathrm{CH}^{+}$lines show velocity components, which can be distinguished by fitting peak profiles onto them when they are not well separated. As interstellar wavelength references, we chose the lines indicating the same velocity as the strongest $\mathrm{CH}$ and $\mathrm{CN}$ components, that is, we assume $\mathrm{C}_{60}$ is most likely to be found in the denser regions of diffuse clouds and in translucent clouds. Table B.1 presents the wavelengths of the interstellar diatomic lines seen by the observer and the associated average radial velocities $\bar{v}_{\text {obs }}$. They were determined by fitting Gaussian profiles to the diatomic lines as well as to the background stellar absorptions whenever it was necessary and possible.

\section{Appendix C: Matrix-isolation spectroscopy of $\mathrm{C}_{60}$ in solid $\mathrm{Ne}$}

The equipment used for measuring the spectrum of $\mathrm{C}_{60}$ isolated in $\mathrm{Ne}$ ice was described in previous studies (e.g., Rouillé et al. 2009). Conditions and parameters specific to the present case are as follows. Fullerene $\mathrm{C}_{60}$ powder (SES Research, Inc., purity $99.5 \%$ ) was heated to $400{ }^{\circ} \mathrm{C}$ and the molecules thus released through an opening in the oven were deposited together with an excess of $\mathrm{Ne}$ atoms (Air Liquide, purity 99.999\%) onto a 2 mm-thick $\mathrm{CaF}_{2}$ substrate (Korth Kristalle $\mathrm{GmbH}$ ) cooled to $7.5 \mathrm{~K}$. The $\mathrm{Ne}$ gas was fed into the vacuum chamber at the mass rate of $5 \mathrm{sccm}$ (standard cubic centimeter per minute) and the matrix deposition process lasted $50 \mathrm{~min}$. Absorption spectra were derived from transmission measurements with a spectrophotometer that scanned wavelengths with a step of $0.2 \mathrm{~nm}$,

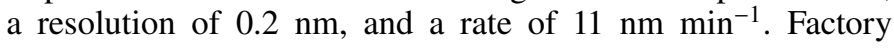
calibration of the wavelength scale was given as accurate to $0.3 \mathrm{~nm}$. 
Table A.1. Observational data sets.

\begin{tabular}{|c|c|c|c|c|}
\hline LOS & Region $(\AA)$ & Resolution & Distance (") & Summed Data Sets \\
\hline \multirow[t]{2}{*}{ HD 76341} & 4000 & 65030 & 0.316 & $\begin{array}{l}\text { ADP.2013-09-27T12_26_24.837.fits, *_25.510.fits, *_25.653.fits, } \\
\text { *_26.350.fits, *_26.810.fits }\end{array}$ \\
\hline & 6000 & 74450 & 0.316 & $\begin{array}{l}\text { ADP.2013-09-27T12_26_24.790.fits, *_24.947.fits, *_24.960.fits, } \\
\text { *_25.710.fits, *_26.303.fits }\end{array}$ \\
\hline \multirow[t]{2}{*}{ HD 136239} & 4000 & 77810 & 0.423 & ADP.2013-09-27T17_01_53.550.fits, *_53.990.fits \\
\hline & 6000 & 74450 & 0.305 & $\begin{array}{l}\text { ADP.2013-09-27T12_26_24.710.fits, *_24.900.fits, *_25.030.fits, } \\
* \_25.090 . \text { fits, *_25.723.fits, *_26.530.fits, *_26.593.fits }\end{array}$ \\
\hline \multirow[t]{2}{*}{ HD 148379} & 4000 & 77810 & 0.639 & $\begin{array}{l}\text { ADP.2013-09-27T17_10_35.883.fits, *_35.897.fits, *_36.043.fits, } \\
* \text { *36.157.fits, *_36.217.fits, *_36.503.fits, *_36.650.fits, } \\
\text { *_36.777.fits, *_36.930.fits, *_37.050.fits }\end{array}$ \\
\hline & 6000 & 87410 & 0.613 & $\begin{array}{l}\text { ADP.2013-09-27T17_10_35.643.fits, *_35.723.fits, *_35.750.fits, } \\
\text { *_35.890.fits, *_36.297.fits, *_36.343.fits, *_36.517.fits, } \\
\text { *_36.750.fits, *_36.797.fits, *_37.257.fits }\end{array}$ \\
\hline \multirow[t]{2}{*}{ HD 167264} & 4000 & 71050 & 1.23 & $\begin{array}{l}\text { ADP.2016-06-13T12_13_59.824.fits, *.825.fits, *.826.fits, *.828.fits, } \\
* .830 . \text { fits, *.832.fits, *.834.fits, *.835.fits, *.837.fits, *.842.fits, } \\
* .847 . \text { fits, *.851.fits }\end{array}$ \\
\hline & 6000 & 74450 & 0.383 & $\begin{array}{l}\text { ADP.2013-09-27T13_08_45.867.fits, *_45.880.fits, *_45.920.fits, } \\
\text { *_45.993.fits, *_46.000.fits, *_46.007.fits, } \\
\text { *_46.013.fits, *_46.113.fits, *_46.160.fits }\end{array}$ \\
\hline \multirow[t]{2}{*}{ HD 168625} & 4000 & 65030 & 4.46 & ADP.2017-10-24T09_50_31.392.fits, *.394.fits, *.396.fits, *.412.fits \\
\hline & 6000 & 74450 & 0.027 & ADP.2017-10-24T09_50_31.415.fits, *.437.fits \\
\hline \multirow[t]{2}{*}{ HD 169454} & 4000 & 71050 & 0.511 & ADP.2016-09-06T06_22_18.210.fits, *.232.fits, *.234.fits \\
\hline & 6000 & 66320 & 0.451 & $\begin{array}{l}\text { ADP.2017-10-20T16_04_39.935.fits, *.946.fits, *.947.fits, *.948.fits, } \\
* .951 . f i t s, * .952 . f i t s, * .955 . f i t s, * .969 . f i t s, * .981 . f i t s, * .982 . f i t s\end{array}$ \\
\hline \multirow[t]{2}{*}{ HD 183143} & 4000 & 71050 & 0.827 & ADP.2018-10-11T06_09_48.104.fits \\
\hline & 6000 & 66320 & 0.31 & ADP.2013-09-26T22_20_55.787.fits, *.807.fits, *.820.fits \\
\hline
\end{tabular}

Notes. Asterisks replace common parts in successive file names. The ESO reprocessed the UVES data sets and stacked them when adequate during the preparation of this manuscript. The original data sets are still valid.

Table B.1. Wavelengths and average radial velocities in the observer rest frame for interstellar molecules.

\begin{tabular}{lllllllll}
\hline \hline LOS & $\lambda_{\mathrm{CN}}(\AA)$ & $\lambda_{\mathrm{CN}}(\AA)$ & $\lambda_{\mathrm{CN}}(\AA)$ & $\lambda_{\mathrm{CH}}(\AA)$ & $\lambda_{\mathrm{CH}^{+}}(\AA)$ & $\lambda_{\mathrm{CH}^{+}}(\AA)$ & $\lambda_{\mathrm{CH}}(\AA)$ & $\bar{v}_{\mathrm{obs}}\left(\mathrm{km} \mathrm{s}^{-1}\right)$ \\
\hline HD 76341 & $\ldots$ & 3875.05 & $\ldots$ & 3886.85 & 3958.14 & 4233.03 & 4300.81 & +33.9 \\
& $\ldots$ & $(+0.44)$ & $\ldots$ & $(+0.44)$ & $(+0.43)$ & $(+0.48)$ & $(+0.50)$ & \\
HD 136239 & 3873.51 & 3874.12 & 3875.27 & 3885.91 & 3957.20 & 4232.02 & 4299.77 & -38.1 \\
& $(-0.49)$ & $(-0.49)$ & $(-0.50)$ & $(-0.50)$ & $(-0.51)$ & $(-0.53)$ & $(-0.54)$ & \\
HD 148379 & 3873.37 & 3873.97 & $\ldots$ & 3885.77 & 3957.06 & 4231.87 & 4299.61 & -49.0 \\
& $(-0.63)$ & $(-0.64)$ & $\ldots$ & $(-0.64)$ & $(-0.65)$ & $(-0.68)$ & $(-0.70)$ & \\
HD 167264 & $\ldots$ & $\ldots$ & $\ldots$ & 3886.03 & 3957.30 & 4232.13 & 4299.89 & -29.9 \\
& $\ldots$ & $\ldots$ & $\ldots$ & $(-0.38)$ & $(-0.41)$ & $(-0.42)$ & $(-0.42)$ & \\
HD 168625 & $\ldots$ & 3874.79 & $\ldots$ & 3886.61 & $\ldots$ & $\ldots$ & 4300.54 & +15.1 \\
& $\ldots$ & $(+0.18)$ & $\ldots$ & $(+0.20)$ & $\ldots$ & $\ldots$ & $(+0.23)$ & \\
HD 169454 & 3874.12 & 3874.73 & 3875.89 & 3886.53 & 3957.81 & 4232.68 & 4300.46 & +9.2 \\
& $(+0.12)$ & $(+0.12)$ & $(+0.12)$ & $(+0.12)$ & $(+0.10)$ & $(+0.13)$ & $(+0.15)$ & \\
HD 183143 & 3874.29 & 3874.90 & 3876.06 & 3886.70 & 3957.98 & 4232.86 & 4300.63 & +22.1 \\
& $(+0.29)$ & $(+0.29)$ & $(+0.29)$ & $(+0.29)$ & $(+0.27)$ & $(+0.31)$ & $(+0.32)$ & \\
\hline
\end{tabular}

Notes. Numbers between parentheses are shifts from rest values given by Hobbs et al. (2009). Three- $\sigma$ error smaller than $0.01 \AA$ for $75 \%$ of the fitted wavelengths. One- $\sigma$ error greater than $0.01 \AA$ in one case only. 